\title{
ARTICLES
}

\section{Inclusive and exclusive decays of $B$ mesons to final states including charm and charmonium mesons}

D. Bortoletto, D. N. Brown, J. Dominick, R. L. McIlwain, D. H. Miller, M. Modesitt, E. I. Shibata, S. Schaffner, and I. P. J. Shipsey Purdue University, West Lafayette, Indiana 47907

M. Battle, H. Kroha, K. Sparks, E. H. Thorndike, and C.-H. Wang University of Rochester, Rochester, New York 14627

M. Goldberg, T. Haupt, N. Horwitz, V. Jain, G. C. Moneti, Y. Rozen, P. Rubin, T. Skwarnicki, V. Sharma, S. Stone, M. Thusalidas, W.-M. Yao, and G. Zhu Syracuse University, Syracuse, New York 13244

A. V. Barnes, J. Bartelt, S. E. Csorna, T. Letson, and M. D. Mestayer Vanderbilt University, Nashville, Tennessee 37235

J. Alexander, M. Artuso, C. Bebek, K. Berkelman, D. Besson, T. E. Browder, D. G. Cassel, E. Cheu, D. M. Coffman, P. S. Drell, R. Ehrlich, R. S. Galik, M. Garcia-Sciveres, B. Geiser, B. Gittelman, S. W. Gray, A. M. Halling, D. L. Hartill, B. K. Heltsley, K. Honscheid, J. Kandaswamy, N. Katayama, D. L. Kreinick, J. D. Lewis, G. S. Ludwig, J. Masui, J. Mevissen, N. B. Mistry, S. Nandi, C. R. Ng, E. Nordberg, C. O’Grady, D. Peterson, M. Pisharody,

D. Riley, M. Sapper, M. Selen, A. Silverman, H. Worden, and M. Worris Cornell University, Ithaca, New York 14853

P. Avery, A. Freyberger, L. Garren, J. Rodriguez, and J. Yelton University of Florida, Gainesville, Florida 32611

K. Kinoshita, F. Pipkin, M. Procario, R. Wilson, J. Wolinski, D. Xiao, and Y. Zhu Harvard University, Cambridge, Massachusetts 02138

A. J. Sadoff

Ithaca Collega, Ithaca, New Yrok 14850

R. Ammar, P. Baringer, D. Coppage, R. Davis, P. Haas, M. Kelly, N. Kwak, H. Lam, and S. Ro

University of Kansas, Lawrence, Kansas 66045

Y. Kubota, J. K. Nelson, D. Perticone, R. Poling, and S. Schrenk University of Minnesota, Minneapolis, Minnesota 55455

M. S. Alam, I. J. Kim, W. C. Li, B. Nemati, V. Romero, C. R. Sun, P.-N. Wang, and M. M. Zoeller

State University of New York at Albany, Albany, New York 12222

G. Crawford, R. Fulton, K. K. Gan, T. Jensen, D. R. Johnson, H. Kagan,

R. Kass, R. Malchow, F. Morrow, J. Whitmore, and P. Wilson Ohio State University, Columbus, Ohio 43210

(CLEO Collaboration)

(Received 29 July 1991)

We have studied hadronic decays of $B$ mesons. We report measurements of exclusive branching ratios of several charm decay modes of $B$ mesons to final states with a $D$ or $D^{*}$ and one to three charged pions or a charged $\rho$ and to final states with a $\psi$ or $\psi^{\prime}$, a kaon, and up to two charged pions. We have also mea- 
sured inclusive branching ratios for $B$ decays to $D$ and $D^{*}$ and the spectra of these particles in $B$ decays. The total charm content in $B$ decay is found to be $(101 \pm 12) \%$. The branching ratios and spectra are compared to form-factor models. We extract the parameters $a_{1}$ and $a_{2}$ of the model of Bauer, Stech, and Wirbel and the $D_{S}$ decay constant. The masses of the $\bar{B}^{0}$ and $B^{-}$mesons are measured. The mass difference between $\bar{B}^{0}$ and $B^{-}$is found to be $-0.4 \pm 0.6 \pm 0.5 \mathrm{MeV} / c^{2}$.

PACS number(s): $13.25 .+\mathrm{m}, 14.40 . \mathrm{Jz}$

\section{INTRODUCTION}

Kinematic reconstruction of exclusive decays provides the only means of measuring the masses of the neutral and charged $B$ mesons. A precision measurement of the mass difference may give a better understanding of the electromagnetic and strong interactions between a heavy quark and a light quark. In this paper, we present new measurements of the $\bar{B}^{0}$ and $B^{-}$masses and of the $\bar{B}^{0}-B^{-}$mass difference as well as exclusive branching ratios, including those of some heretofore unreported modes. Exclusive hadronic decays rates of $B$ mesons can also provide important tests of models of $B$ meson decay [1-5]. Inclusive measurement of the total charm content in $B$ decays provides confirmation that $b \rightarrow c$ spectator processes dominate. Measurements of the momentum spectra of $D$ and $D^{*}$ mesons produced in $B$ decay can be compared to theoretical models of $B$ decay [6].

All $B$ meson decays considered here have at least one charmed quark in the final state and result from the dominant $b \rightarrow c$ transition. When the reactions proceed through the chain $b \rightarrow c W^{-}, W^{-} \rightarrow \overline{c s},{ }^{1}$ there are two charmed quarks in the final state that can hadronize either as two charmed particles or as charmonium (for example, $\psi$ or $\left.\psi^{\prime}\right)$. Otherwise, there is only one charmed particle in the final state.

\section{DETECTOR AND DATA SAMPLES}

The data sample used in this study was collected in 1987 using the CLEO detector at the Cornell Electron Storage Ring (CESR). The $B$ mesons result from the decays of the $\Upsilon(4 S)$ resonance in $e^{+} e^{-}$annihilations. The data consist of $212 \mathrm{pb}^{-1}$ at the $\Upsilon(4 S)$ resonance containing $240000 B \bar{B}$ pairs and $102 \mathrm{pb}^{-1}$ at energies below $B \bar{B}$ threshold. The CLEO detector, described in detail elsewhere [7], underwent major improvements to the central tracking system in 1986 [8]. Charged particle tracking is done inside a superconducting solenoid of radius $1.0 \mathrm{~m}$ which produces a $1.0 \mathrm{~T}$ magnetic field. Three nested cylindrical drift chambers measure momenta and specific ionization $(d E / d x)$ of charged particles. The momentum resolution achieved by this system is $(\delta p / p)^{2}=(0.23 \% p)^{2}+(0.7 \%)^{2}$, where $p$ is in $\mathrm{GeV} / c$. The main drift chamber measures $d E / d x$ to $6.5 \%$ accuracy. Photons are detected in calorimeters that cover $47 \%$ of the solid angle. The photon energy resolution is

\footnotetext{
${ }^{1}$ For any process described herein, the charge-conjugate process is implied as well.
}

$\sigma_{E} / E=21 \% / \sqrt{E}$, where $E$ is in $\mathrm{GeV}$, and the angular resolution is $10 \mathrm{mrad}$.

All events under consideration in this analysis pass our hadronic event-selection criteria $[9,10]$. Briefly, for this analysis there are four primary requirements.

(i) The ratio of the number of bad track candidates (those with no $z$ fit or high residuals and those that do not point to the interaction region) to the number of good tracks is required to be less than 1.15 .

(ii) A minimum value for visible energy (charged tracks plus energy deposits in the calorimeter not matched to charged tracks) of $30 \%$ of the center-of-mass energy is demanded.

(iii) The total energy measured in the electromagnetic calorimeter is required to be between 0.5 and $7.0 \mathrm{GeV}$.

(iv) Since the final-state hadron momenta should balance, the ratio of Fox-Wolfram moments [11] $R_{1} \equiv H_{1} / H_{0}$ for charged particles is required to be less than 0.45 .

The efficiency of these hadronic event-selection cuts for $B \bar{B}$ events is measured to be $96 \%$. The efficiency of the trigger for $B \bar{B}$ events has been found in Monte Carlo simulations to be $98 \%$.

\section{INCLUSIVE CHARMED-PARTICLE RATES AND SPECTRA}

\section{A. Charmed mesons}

In order to measure the inclusive spectra, we search for charmed mesons in decay modes with clean signatures in invariant-mass distributions. We define $x=p / p_{\max }$, where $p$ is the measured particle momentum and $p_{\max }=\sqrt{E_{\text {beam }}^{2}-M^{2}}$ is the maximum possible momentum the particle could have if pair produced in continuum $e^{+} e^{-}$annihilations. Because at the $\Upsilon(4 S) B$ mesons are produced nearly at rest, the $B$ daughters satisfy $x<0.5$. Signals for charmed mesons are sought in the following decay modes:

$$
\begin{aligned}
D^{0} \rightarrow K^{-} \pi^{+}, D^{+} \rightarrow K^{-} \pi^{+} \pi^{+}, \quad D^{*+} & \rightarrow D^{0} \pi^{+} \\
& \longleftrightarrow K^{-} \pi^{+} .
\end{aligned}
$$

In order to reduce combinatoric background, particles are required to have specific ionization measurements within two standard deviations of that expected for their assignment in the formation of the candidate $D$. The inclusive rates are obtained by finding the number $N_{\text {on }}$ of $D$ 's and $\bar{D}$ 's in a particular mode in data taken at the $\Upsilon(4 S)$ energy and the number $N_{\text {off }}$ at an energy below $B \bar{B}$ threshold. We subtract, scaling by the ratio of luminosities and accounting for the $1 / s$ dependence of the nonresonant $e^{+} e^{-}$annihilation cross section. If $D^{z}$ is a 
charmed meson of some type, then we measure the number of $D^{z \text { 's }}$ and $\bar{D}^{2}$ 's resulting from $\Upsilon(4 S)$ decays as

$$
N^{z}=N_{\text {on }}^{z}-\frac{\mathcal{L}_{\text {on }}}{\mathcal{L}_{\text {off }}} \frac{s_{\text {off }}}{s_{\text {on }}} N_{\text {off }}^{z} .
$$

$D^{z}$ and $\bar{D}^{z}$ mesons can be produced in $\Upsilon(4 S)$ events either by the decay of $B$ mesons or by the direct decay of the $\Upsilon(4 S)$. Therefore, the number produced is

$$
\begin{aligned}
N_{0}^{z}=N_{\Upsilon(4 S)}\{ & {\left[f_{ \pm}\left(\mathcal{B}\left(B^{-} \rightarrow D^{z}\right)+\mathcal{B}\left(B^{+} \rightarrow D^{z}\right)\right)\right.} \\
& \left.+f_{0}\left(\mathcal{B}\left(\bar{B}^{0} \rightarrow D^{z}\right)+\mathcal{B}\left(B^{0} \rightarrow D^{z}\right)\right)\right] \\
& +\left(1-f_{B \bar{B}}\right) \mathcal{B}\left(\Upsilon(4 S) \rightarrow D^{z}\right) \\
& + \text { charge conjugate } D \text { 's }\},
\end{aligned}
$$

where $f_{B \bar{B}}$ is the fraction of $\Upsilon(4 S)$ decays to $B \bar{B}, f_{ \pm}\left(f_{0}\right)$ is the fraction of $\Upsilon(4 S)$ events that contain a $B^{+} B^{-}$ $\left(B^{0} \bar{B}^{0}\right)$ pair, $f_{0}+f_{ \pm}=f_{B \bar{B}}$, and $\mathcal{B}(x)$ is the branching ratio in decay mode $x$.

To calculate branching ratios from measured yields, one must know or assume values for $f_{ \pm} / f_{0}$ and $f_{B \bar{B}}$. In previous publications, the CLEO Collaboration obtained $f_{ \pm} / f_{0}$ by assuming a $p^{3}$ dependence based on a phasespace argument for $\Upsilon(4 S) \rightarrow B \bar{B}$. However, because the wavelength of the $B$ is not large with respect to the size of the $\Upsilon(4 S)$, simple threshold calculations are not sufficient, and in order to predict the fractions, one must have detailed knowledge of the $\Upsilon(4 S)$ wave function as well as precise measurements of the $B$ masses [12]. In the absence of detailed knowledge, we assume $f_{ \pm} / f_{0}=1$ throughout this paper. Also, as in the past, we assume [13] $f_{B \bar{B}}=1$, implying $\mathscr{B}\left(B \rightarrow D^{z} X\right)=N^{2} / 2 N_{\Upsilon(4 S)}$.

The signals for $D^{0}, D^{+}$, and $D^{*+}$ in the modes studied are shown for both the $\Upsilon(4 S)$ and continuum data samples in Fig. 1. In each mode, the data are divided into several bins in $x$, and the invariant-mass distributions for $D$ candidates from the $\Upsilon(4 S)$ and continuum data samples are each fit to a signal function determined for each $x$ bin by Monte Carlo studies and the background form $y=\exp \left(a+b m+c m^{2}\right)$ with adjustable coefficients $a, b$, and $c$. For the $D^{+}$, the signal function is a single Gaussian. However, $d E / d x$ measurements are in many cases not sufficient to differentiate between the $K$ and the $\pi$ in $D^{0}$ decay. Therefore, to account for the $K^{-} \pi^{+}$combination incorrectly assigned the $K^{+} \pi^{-}$hypothesis, the signal function includes a second Gaussian which is about three times as wide and with the mean, width, and area fixed by Monte Carlo simulations relative to those of the right assignment combination. We also include a bifurcated Gaussian at a slightly lower mass determined by Monte Carlo studies representing the contribution from the decay $D^{0} \rightarrow K^{-} K^{+}$, where one of the kaons is misidentified. The area is fixed to that for the $K \pi$ decays accounting for the relative efficiencies and the relative branching ratios [14]. Because the rate for $D^{0} \rightarrow K^{-} \pi^{+} \pi^{0}$ is more than three times that for $D^{0} \rightarrow K^{-} \pi^{+}$, we also observe a large but wide "satellite peak" well below the $D^{0}$ mass from $K^{-} \pi^{+} \pi^{0}$ decays where the $\pi^{0}$ is not observed. In order to allow a good fit of the background, we include a function determined by

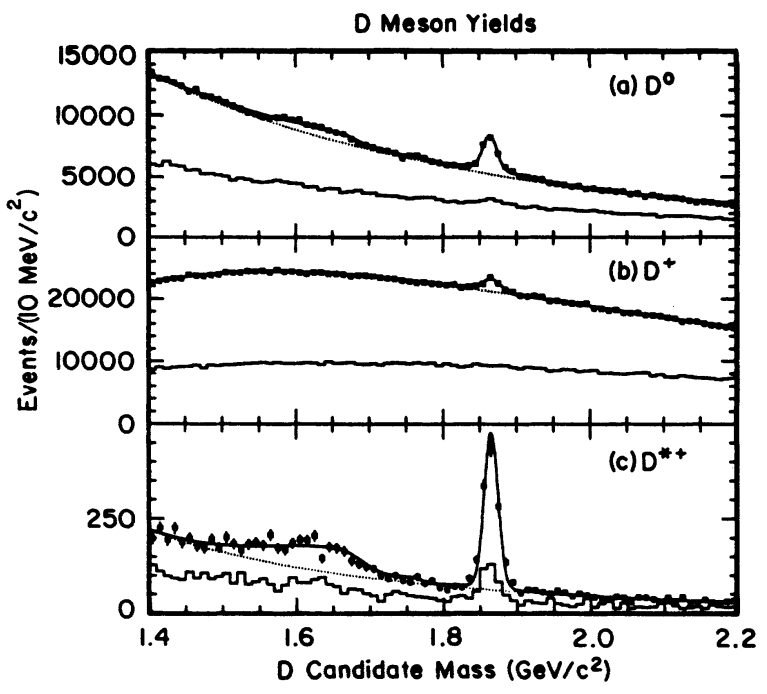

FIG. 1. Invariant-mass distributions showing $D$ meson production in the $\Upsilon(4 S)$ energy region. The points show results from the $\Upsilon(4 S)$, and the histograms show the continuum results scaled for the difference in luminosity and cross section. (a) $D^{0} \rightarrow K^{-} \pi^{+}$; (b) $D^{+} \rightarrow K^{-} \pi^{+} \pi^{+} ;$and (c) $D^{*+} \rightarrow D^{0} \pi^{+}$, $D^{0} \rightarrow K^{-} \pi^{+}$. We have required $x<0.5$ in all three and $R_{2}<0.3$ in $\mathrm{a}$ and $\mathrm{b}$. The dotted line shows the exponential background function.

Monte Carlo simulation for the $K^{-} \pi^{+} \pi^{0}$ contribution in each $x$ bin. However, because the distribution is wide and other processes such as $D^{+} \rightarrow K^{-} \pi^{+} \pi^{+}$also contribute, and due to uncertainties in the resonant substructure of the decay, we allow the normalization of this component to float.

To find the $D^{*+}$ yields, we fit the $D^{0}$ candidate mass distribution requiring the $\left(K^{-} \pi^{+}\right) \pi^{+}-\left(K^{-} \pi^{+}\right)$mass difference to be within $2.0 \mathrm{MeV} / \mathrm{c}^{2}$ of the known mass difference between $D^{*}$ and $D$. The rms resolution for the mass difference is $0.7 \mathrm{MeV} / c^{2}$. Because the sign of the slow pion determines the sign of the kaon, eliminating $K \pi$ misassignment for a real $D^{0}$, a single narrow Gaussian is nearly sufficient to describe the $D^{0}$ mass distribution. However, a small $(<10 \%)$ component is described by a second wider Gaussian arising from events with poorly measured tracks. The functions for $K^{-} \pi^{+} \pi^{0}$ and $K^{-} K^{+}$are included in the fit, but their contributions are smaller as a result of the determination of the kaon charge and the mass difference cut.

In each $x$ bin, the $B \bar{B}$ yield is found by subtracting the yield in the continuum data sample from that for the $\Upsilon(4 S)$ data set, scaled by the ratios of continuum annihilation cross section and integrated luminosity. Because at the $\Upsilon(4 S)$ energy the continuum cross section is three times the $B \bar{B}$ cross section, it is useful to suppress the continuum contribution before subtraction. We take advantage of the fact that the $B$ mesons are produced nearly at rest so their decay products are distributed spherically, while continuum events are jetlike. We describe the event shape by the Fox-Wolfram parameter ratio [11] $R_{2}=H_{2} / H_{0}$ which is near zero for purely spherical 
events and approaches unity for collinear events. Requiring $R_{2}<0.3$ eliminates $60 \%$ of the continuum and is $90 \%$ efficient for $B \bar{B}$ events. This cut is not used for the $D^{*+}$ measurements because the mass difference cut virtually eliminates the combinatoric background. We further reduce random combinations from continuum events in the $D^{0} \rightarrow K^{-} \pi^{+}$sample by requiring $\cos \theta_{K}>-0.8$, where $\theta_{K}$ is the angle between the $D^{0}$ candidate momentum in the laboratory frame and the $K$ momentum in the $K \pi$ rest frame.

The efficiency for finding a $D^{*+}$ is a strong function of the momentum of the slow daughter pion. The distribution of pion momenta is determined by the $D^{*+}$ momentum spectrum and polarization. We define the polarization angle $\theta_{H}$ to be the angle between the $D^{*+}$ momentum and the momentum of the daughter pion in the rest frame of the $D^{*+}$. In each momentum bin, we further divide the data into bins in $\cos \theta_{H}$. We then perform a binned likelihood fit of the continuum-subtracted yields in each momentum bin over $\cos \theta_{H}$ to the function

$$
f_{x}\left(\theta_{H}\right)=N_{x} \epsilon_{x}\left(\theta_{H}\right) \frac{\beta \cos ^{2} \theta_{H}+(1-\beta) \sin ^{2} \theta_{H}}{1+\frac{1}{3} \beta},
$$

where $N_{x}$ is the efficiency corrected yield in each momentum bin, the efficiency function $\epsilon_{x}\left(\theta_{H}\right)$ is determined in each $x$ bin from Monte Carlo studies and fit to a polynomial function, and the polarization parameter $\beta$ ranges from -1 to 1 . Figure 2 shows the continuum-subtracted $D^{*+}$ yield from $\Upsilon(4 S)$ events as a function of $\cos \theta_{H}$ and $x$ with the best fits to $f_{x}$. Figure 3 compares the measured values of the polarization parameter $\beta$ to the theoretical predictions of Wirbel and $\mathrm{Wu}$ [6]. The data and model agree well; however, the errors on the data points are quite large.

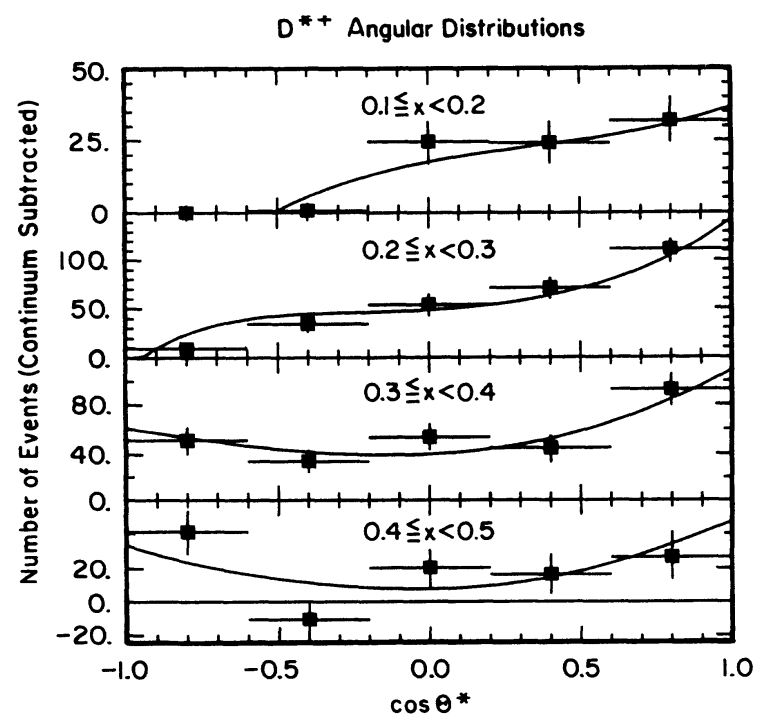

FIG. 2. Continuum-subtracted $D^{*+}$ yield from $\Upsilon(4 S)$ events as a function of the $D^{*}$ decay angle and the scaled momentum $x=p / \sqrt{E_{\text {beam }}^{2}-m^{2}}$. The curves show the result of the best fit to $f_{x}$ given in Eq. (1).

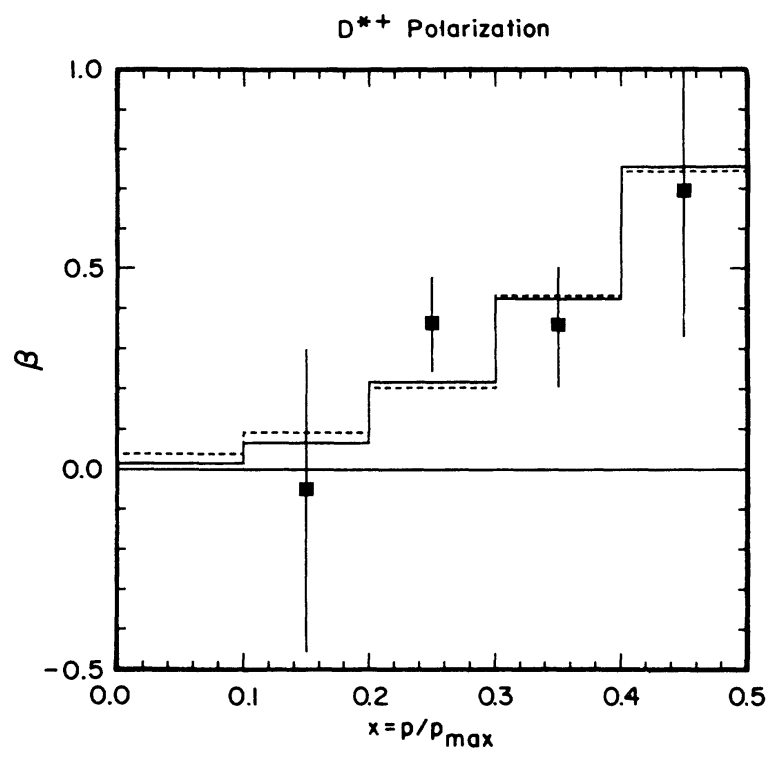

FIG. 3. The measured values of the polarization parameter $\beta$ are compared to the theoretical predictions. The points show the data. The solid line shows the free-quark model prediction of Ref. [6], and the dashed line that of the semiphenomenological model.

We sum the efficiency-corrected yields in all $x$ bins to find measured product branching ratios:

$$
\begin{aligned}
& \mathscr{B}\left(B \rightarrow D^{0} X\right) \mathcal{B}\left(D^{0} \rightarrow K^{-} \pi^{+}\right)=0.0233 \pm 0.0012 \pm 0.0014, \\
& \begin{aligned}
\mathcal{B}\left(B \rightarrow D^{+} X\right) \mathcal{B}\left(D^{0} \rightarrow K^{-} \pi^{+} \pi^{+}\right) \\
\quad=0.0226 \pm 0.0030 \pm 0.0018, \\
\mathcal{B}\left(B \rightarrow D^{* *} X\right) \mathcal{B}\left(D^{*+} \rightarrow D^{0} \pi^{+}\right) \mathcal{B}\left(D^{0} \rightarrow K^{-} \pi^{+}\right) \\
=0.00556 \pm 0.00031 \pm 0.00050 \quad(0.1 \leq x<0.5) ;
\end{aligned}
\end{aligned}
$$

where the first error is statistical and the second is systematic. The systematic error results from the uncertainty in efficiencies and fitting procedures.

To find the total decay rates, it is necessary to divide by the measured $D$ branching ratios in the modes studied. We choose to use the branching ratios measured by the Mark III Collaboration $[15,16]$ for $D^{0}, D^{+}$, and $D^{*}$. We use world average values [17] for $\psi$ and $\psi^{\prime}$. Table I lists the charm branching ratios used in this paper as well as the efficiencies for the modes used in the inclusive analysis. The $D^{*+}$ efficiency is averaged over the observed momentum and decay-angle distributions.

Low efficiency prevents us from measuring the $D^{*}$ yield for $x<0.1$. However, we can estimate the contribution of this momentum range using the assumptions that $f_{0}=f_{ \pm}$and that all $B \rightarrow D X$ decays can be described by the simple spectator model. Therefore, one expects that the direct decay rates of $B \rightarrow D^{0}$ and $B \rightarrow D^{+}$should be nearly equal, as should those for $B \rightarrow D^{* 0}$ and $B \rightarrow D^{*+}$. Also, the $D$ spectra from the decay chain $B \rightarrow D^{*} \rightarrow D$ should have approximately the same shape for $D^{*} \rightarrow D \pi$ and $D^{*} \rightarrow D \gamma$. Because $\mathcal{B}\left(D^{*+} \rightarrow D^{0}\right)$ does not equal $\mathcal{B}\left(D^{* 0} \rightarrow D^{+}\right)$, in the difference of the $D^{0}$ and $D^{+}$ 
TABLE I. Charm branching ratios and inclusive efficiencies.

\begin{tabular}{ccc}
\hline \hline Charm decay mode & Branching ratio (\%) & Efficiency $(\%)$ \\
\hline$D^{0} \rightarrow K^{-} \pi^{+}$ & $4.2 \pm 0.6$ & 53 \\
$D^{0} \rightarrow K^{-} \pi^{+} \pi^{+} \pi^{-}$ & $9.1 \pm 1.1$ & \\
$D^{0} \rightarrow \bar{K}^{0} \pi^{+} \pi^{-}$ & $6.4 \pm 1.1$ & \\
$D^{+} \rightarrow K^{-} \pi^{+} \pi^{+}$ & $9.1 \pm 1.4$ & 41 \\
$D^{+} \rightarrow \bar{K}^{0} \pi^{+}$ & $3.2 \pm 0.5$ & \\
$D^{*+} \rightarrow D^{0} \pi^{+}$ & $57 \pm 6$ & $28^{\mathrm{a}}$ \\
$\psi \rightarrow l^{+} l^{-}$ & $6.9 \pm 0.9$ & \\
$\psi^{\prime} \rightarrow l^{+} l^{-}$ & $0.9 \pm 0.2$ & \\
$\psi^{\prime} \rightarrow \psi \pi^{+} \pi^{-}$ & $33 \pm 3$ & \\
$D_{S}^{+} \rightarrow \phi \pi^{+}$ & $3.0 \pm 1.1$ & \\
\hline \hline
\end{tabular}

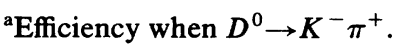

momentum spectra the direct $D$ contribution is canceled, and the resulting distribution should be similar to the $D$ spectrum from $B \rightarrow D^{*}$ decays. Since the pion carries very little of the $D^{*}$ momentum, we estimate that in $B$ decay, the fraction of $D$ from $D^{*}$ with $x<0.1$ is equal to the fraction of $D^{*}$ with $x<0.1$; that is,

$$
\begin{aligned}
\frac{N^{*}(x<0.1)}{N^{*}(x \geq 0.1)} & \approx \frac{N^{0}(x<0.1)-N^{+}(x<0.1)}{N^{0}(x \geq 0.1)-N^{+}(x \geq 0.1)} \\
& =0.086 \pm 0.040 .
\end{aligned}
$$

Figure 4 shows the inclusive spectra after continuum subtraction and accounting for $D$ branching ratios [19] and for efficiencies obtained from Monte Carlo simulations.

Table II lists the measured inclusive branching ratios along with prior measurements by the CLEO [18] and

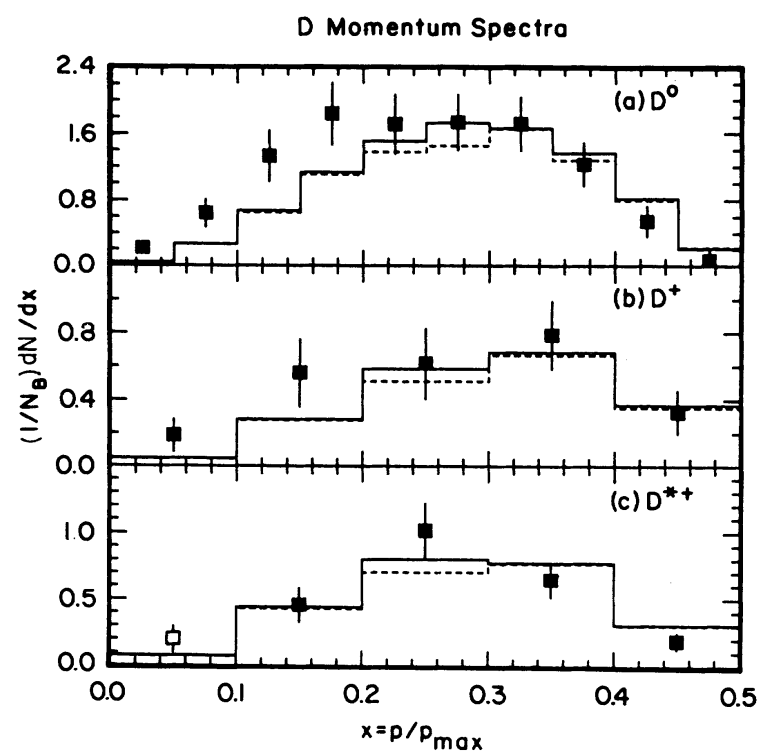

FIG. 4. Momentum spectra for $D^{0}, D^{+}$, and $D^{*+}$ mesons in $B$ meson decays. The solid squares show the data. The open square is derived from the difference of the $D^{0}$ and $D^{+}$spectra. The histograms show the predictions of Ref. [6]. The solid line shows the free-quark model prediction, and the dashed line shows the semiphenomenological model.
ARGUS [19] Collaborations and the predictions of the free-quark (I) and semiphenomenological (II) models of Ref. [6] assuming that the $B$ lifetime is $\tau_{B}=1.2 \mathrm{ps}$ and the $b \rightarrow c$ Cabibbo-Kobayashi-Maskawa (CKM) matrix element is $\left|V_{c b}\right|=0.05$. The first error on the new measurements is statistical, the second is systematic, and the third results from $D$ branching ratios. For the older measurements the first error includes both statistical and systematic errors, and the second accounts for the uncertainty in $D$ branching ratios.

Figure 4 also shows the form-factor model predictions of Wirbel and Wu [6]. They expand upon the method of Bauer, Stech, and Wirbel used for semileptonic decays [20] and two-body decays [1]. Under the factorization hypothesis, the weak-interaction matrix element for spectator decays of $B$ mesons to $D$ or $D^{*}$ is described by a current-current interaction:

$$
M_{W} \sim V_{c b}\left\langle f_{1} \bar{f}_{2}\left|\left(\bar{f}_{1} f_{2}\right)^{\mu}\right| 0\right\rangle\left\langle D / D^{*}\left|(\bar{c} b)_{\mu}\right| B\right\rangle,
$$

where $f_{1} \bar{f}_{2}$ is a fermion-antifermion pair (e.g., $d \bar{u}, e^{-} \bar{v}$ ). The heavy-quark term, $\left\langle D / D^{*}\left|(\bar{c} b)_{\mu}\right| B\right\rangle$, is calculated using a form-factor model. For the hadronic decays, two models are used to describe the polarization of the recoiling system. In Figure 4, the prediction of the free-quark model is shown with the solid line and that of a semiphenomenological model similar to that of Pietschmann and Rupertsberger [21] is shown with the broken line. The theoretically predicted spectra agree well with the observations at high momenta; however, there are significant excesses of the data over the predictions at low $D$ momenta. This observation may indicate production of high mass $D$ resonances or fragmentation between the $c$ quark and the spectator quark.

\section{B. Total $B \rightarrow c$ branching ratio}

The inclusive branching ratios for decay into charged mesons, averaged over the mixture of $\bar{B}^{0}$ and $B^{-}$at the $\Upsilon(4 S)$, are given in Table III. The cascade decays from the $D^{*+}$ are not subtracted from $D^{0}$ or $D^{+}$rates. Neither are $\psi^{\prime}$ cascades subtracted from the inclusive $\psi$ rate. The inclusive $B \rightarrow D_{S}$ branching ratio reported in Ref. 
TABLE II. Inclusive $B \rightarrow D$ branching ratios (\%).

\begin{tabular}{cccr}
\hline & $B \rightarrow D^{0} X$ & $B \rightarrow D^{+} X$ & $B \rightarrow D^{*+} X$ \\
\hline CLEO 1987 & $55.4 \pm 3.0 \pm 3.3 \pm 7.5$ & $24.9 \pm 3.3 \pm 2.0 \pm 3.1$ & $25.2 \pm 1.4 \pm 2.5 \pm 4.4$ \\
CLEO 1985 [18] & $49 \pm 7 \pm 7$ & $26 \pm 6 \pm 4$ & $28 \pm 5 \pm 5$ \\
ARGUS [19] & $46.2 \pm 3.6 \pm 2.9 \pm 6.2$ & $23.0 \pm 3.0 \pm 4.4 \pm 2.9$ & $29.7 \pm 2.5 \pm 5.0 \pm 4.1$ \\
Model I & 47.9 & 20.0 & 24.5 \\
Model II & 45.1 & 18.7 & 38.6 \\
\hline \hline
\end{tabular}

[26] has been recalculated to account for the average of the recent CLEO [23] and ARGUS [24] measurements ${ }^{2}$ of $\mathscr{B}\left(D_{s}^{+} \rightarrow \phi \pi^{+}\right)=(3.0 \pm 1.1) \%$ and is shown in Table III. The $\Lambda_{c}$ production rate is measured from inclusive baryon yields in $B$ decay [22].

To find the fraction of charm per $B$ decay, we sum the $D^{0}, D^{+}, D_{S}^{+}, \Lambda_{c}$, and $\psi$ rates. The $\psi$ rate is multiplied by 4 to take into account the fact that there are two charmed quarks per $\psi$ and that there are unseen $c \bar{c}$ states, such as $\chi_{C}$ or $\eta_{C}$. The summed rate is $(101 \pm 6 \pm 10) \%$, where the first error is the combined statistical and systematic errors and the second includes the uncertainty on the $D$ branching ratios. This rate is consistent with naive expectations of approximately $115 \%$ [27], if the $b \rightarrow c$ transition accounts for all $b$-quark decays. The large systematic errors, however, leave considerable room for a $b \rightarrow u$ component.

\section{B MESON RECONSTRUCTION}

To search for $B$ 's, we first identify charmed meson ${ }^{3}$ candidates. In addition to the decay modes considered in the inclusive analysis, we also use $D^{+} \rightarrow \bar{K}^{0} \pi^{+}$, $D^{0} \rightarrow K^{-} \pi^{+} \pi^{+} \pi^{-}$, and $D^{0} \rightarrow \bar{K}^{0} \pi^{+} \pi^{-}$, since we can reject background using additional constraints available in exclusive reconstruction. $\bar{K}^{0}$ is observed through the decay $K_{S}^{0} \rightarrow \pi^{+} \pi^{-}$. As in the inclusive analysis, a $D^{*+}$ is required to have a candidate $D^{*+}-D^{0}$ mass difference within $2.0 \mathrm{MeV} / \mathrm{c}^{2}$ of the known value.

A $\psi$ is detected through its decay to an electron or muon pair. At least one of the tracks in a $\psi$ or $\psi^{\prime}$ candidate is required to satisfy stringent lepton identification criteria [28-30]. The second track must pass more modest identification criteria. Muons are identified by their ability to penetrate iron. Electrons are identified by the magnitude of their energy deposit in the shower counters, by the lateral and longitudinal distributions of this energy

${ }^{2}$ We calculate the average of $\mathcal{B}\left(D_{S}^{+} \rightarrow \phi \pi^{+}\right) /$ $\mathcal{B}\left(D_{S}^{+} \rightarrow \phi l^{+} v\right)$ from the ARGUS and CLEO Collaborations. Since the systematic error in each case is dominated by the similar measurement techniques, we conservatively assume that the systematic errors are fully correlated. We derive $\mathcal{B}\left(D_{S}^{+} \rightarrow \phi \pi^{+}\right)$as in Ref. [23] except that we also combine the measurement of $\mathcal{B}\left(D^{+} \rightarrow \bar{K}^{* 0} l^{+} v\right)$ from Ref. [24] with that of Ref. [25], where the ARGUS measurement is scaled by the value of $\mathcal{B}\left(D^{+} \rightarrow K^{-} \pi^{+} \pi^{+}\right)$used throughout this paper.

${ }^{3}$ Hereinafter we refer to any meson with open or hidden charm as a "charmed meson." deposit, and by specific ionization. The distributions of the invariant mass of the dielectron and dimuon candidates, demonstrating strong $\psi$ signals, are shown in Fig. 5. We observe $\psi^{\prime}$ decay both through the cascade decay, $\psi^{\prime} \rightarrow \psi \pi^{+} \pi^{-}$and via the direct decay to a lepton pair. In both cases, we require the dilepton candidate momentum to be less than $1.6 \mathrm{GeV} / c$, the maximum allowed for $\psi^{\prime}$ coming from $B$ decay. To search for the cascade decay, we first detect $\psi$ via the dilepton decay and then consider the invariant-mass difference between the $\psi$ plus a $\pi^{+} \pi^{-}$ pair and the original $\psi$. We insist that the $\pi^{+} \pi^{-}$invariant mass be between 450 and $580 \mathrm{MeV} / \mathrm{c}^{2}$, which includes $88 \%$ of dipions from $\psi^{\prime} \rightarrow \psi \pi \pi$. The resultant distribution, shown in Fig. 6(a), has peak at $587 \mathrm{MeV} / \mathrm{c}^{2}$, evidence for production of the $\psi^{\prime}$. Figure $6(\mathrm{~b})$ presents the evidence for the direct decay of $\psi^{\prime}$ to dileptons in $B$ decay.

We require the charmed particle candidate mass to be within two standard deviations of its known mass [17]. The expected mass resolutions determined in Monte Car-

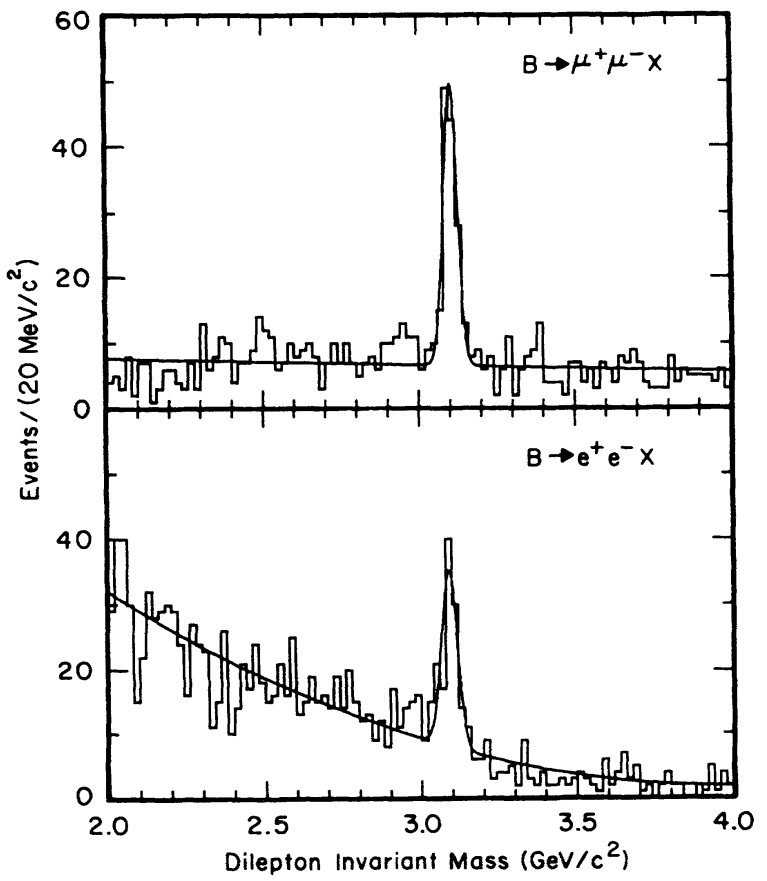

FIG. 5. Dilepton invariant-mass distributions showing $\psi$ meson production in the $\Upsilon(4 S)$ energy region. Both tracks are required to be identified as leptons. The maximum dilepton momentum is required to be less than $2.2 \mathrm{GeV} / c$. 
TABLE III. Inclusive branching ratios (\%).

\begin{tabular}{cc}
\hline$B \rightarrow D^{*+} X$ & $25 \pm 3 \pm 4$ \\
$B \rightarrow D^{0} X$ & $55 \pm 4 \pm 8$ \\
$B \rightarrow D^{+} X$ & $25 \pm 4 \pm 3$ \\
$B \rightarrow D_{S}^{+} X$ & $10 \pm 2 \pm 4$ \\
$B \rightarrow \psi X$ & $1.12 \pm 0.18[17]$ \\
$B \rightarrow \Lambda_{c} X$ & $6.4 \pm 1.1[22]$ \\
\hline
\end{tabular}

lo simulations for various charmed particles are listed in Table IV. In order to improve the measurement of the momentum of the charmed particle, a kinematic fit is performed using the known particle mass as a constraint. Then the candidate is combined with other tracks in the event to form a $B$ candidate. Hadronic resonance (e.g., $\left.K^{*}, \rho\right)$ masses are required to be within $\frac{3}{4} \Gamma$ of their mean value. Neutral pion candidates are formed from two photons, each with an energy greater than $150 \mathrm{MeV}$ and not lying on the projected path of a charged track. A $\pi^{0}$ candidate is required to have an invariant mass within 65 $\mathrm{MeV} / \mathrm{c}^{2}$ of the $\pi^{0}$ mass. Because of the relatively good angle measurement, a kinematic fit constraining the candidate mass to that of the $\pi^{0}$ improves the energy resolution by more than a factor of 2. The $\pi^{0}$ detection efficiency is $10 \%$ for $p_{\pi^{0}}>1 \mathrm{GeV} / c$.

The candidate $B$ has two significant independent kinematic properties: its energy and momentum. We may restrict the value of one and search for enhancement in the distribution of the other. Because the $\Upsilon(4 S)$ is just past the open beauty threshold, it cannot decay into a $b \bar{b}$ final state other than $B^{+} B^{-}$or $B^{0} \bar{B}^{0}$. Therefore, the $B$ energy must be the beam energy, so we consider the $B$ energy
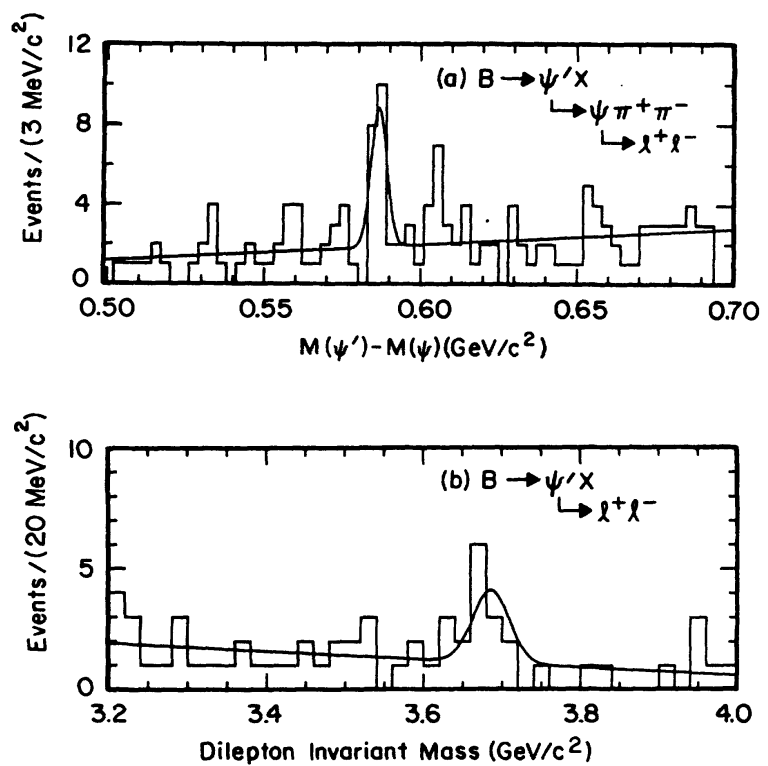

FIG. 6. Invariant-mass distributions showing $\psi^{\prime}$ meson sroduction in the $\Upsilon(4 S)$ energy region. The dilepton momentum is required to be less than $1.6 \mathrm{GeV} / c$. (a) Difference between the reconstructed $\psi^{\prime}$ and $\psi$ masses in the decay $\psi^{\prime} \rightarrow \psi \pi^{+} \pi^{-}, \psi \rightarrow l^{+} l^{-}$. (b) Dilepton mass distribution showing the decay $\psi^{\prime} \rightarrow l^{+} l^{-}$.

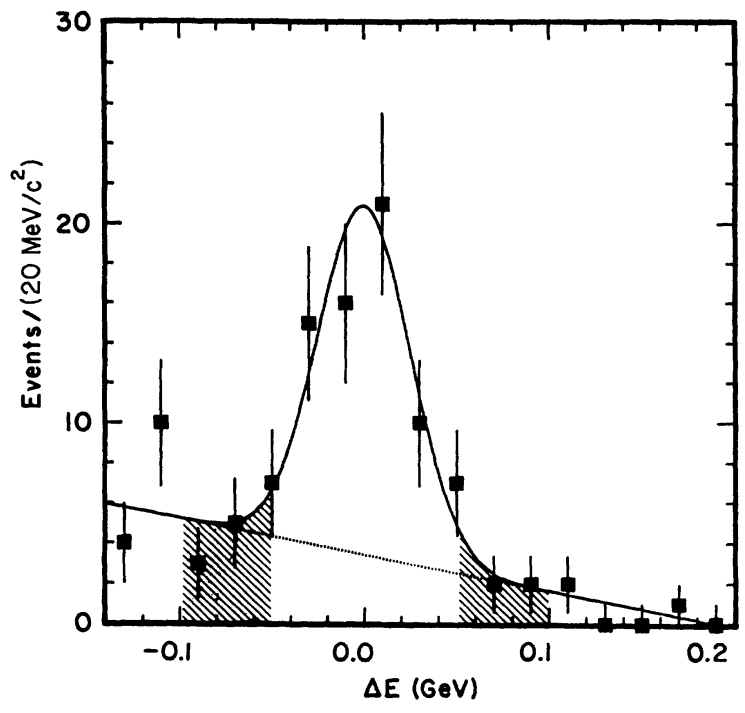

FIG. 7. $\Delta E$ distribution for $\bar{B} \rightarrow D \pi$ and $\bar{B} \rightarrow D^{*} \pi$.

in terms of the difference between the measured energy and the nominal beam energy: $\Delta E=E_{M}-E_{\text {beam }}$. The experimental resolution in $\Delta E$ (Table IV) varies between 15 and $25 \mathrm{MeV}$ for most of the decay modes. The energy resolution is $125 \mathrm{MeV}$ for $\bar{B}^{0} \rightarrow D^{*+} \rho^{-}$as a result of the poor resolution of the $\pi^{0}$ energy.

Because the $B$ energy must be $E_{\text {beam }}$, we compute the $B$ candidate mass from the relation

$$
m^{2}=E_{\text {beam }}^{2}-\mathbf{p}_{B}^{2}
$$

where $\mathbf{p}_{B}^{2}=\left(\Sigma_{i} \mathbf{p}_{i}\right)^{2}$ and $\mathbf{p}_{i}$ is the three-momentum vector of the $i$ th daughter particle. The technique of using $E_{\text {beam }}$ instead of $E_{M}$ improves the resolution in $m\left(\sigma_{m}\right)$ by an order of magnitude. The rms error in $m$ resulting from the error $\sigma_{p}$ in measurement of $\mathbf{p}_{B}$ is

$$
\sigma_{m p}=\frac{\left|\mathbf{p}_{B}\right|}{m} \sigma_{\mathbf{p}} .
$$

Since $\left|\mathbf{p}_{B}\right| / m$ is only 0.06 , this contribution is smaller than the contribution to the error from the $2.0 \mathrm{MeV} \mathrm{rms}$ spread in the beam energy, caused by synchrotron radiation. The net $B$-mass resolution is $\sigma_{m}=2.5 \mathrm{MeV} / c^{2}$. These procedures are similar to those we have used previously [31].

Because the $\Delta E$ resolution is excellent in all modes studied (except $D^{*+} \rho^{-}$), candidates with $|\Delta E|<4 \sigma_{E}$ cannot be real $B$ 's correctly reconstructed with an extra particle or with one particle missing. ${ }^{4}$ Therefore, if we plot the $B$ mass in the signal region $|\Delta E|<2 \sigma_{E}$ and in the sideband region $2 \sigma_{E}<|\Delta E|<4 \sigma_{E}$, the latter provides

\footnotetext{
${ }^{4}$ The one exception is $B \rightarrow D^{*} \pi, D^{*} \rightarrow D \gamma$ in which an insignificant number of events populate the range $-4 \sigma_{E}<\Delta E<-2 \sigma_{E}$ when reconstructed as $D \pi$.
} 
TABLE IV. Experimental parameters in exclusive $B$ reconstruction.

\begin{tabular}{|c|c|c|c|c|c|c|}
\hline $\begin{array}{c}B \text { decay } \\
\text { mode }^{\mathrm{a}}\end{array}$ & $\begin{array}{l}\text { Charm } \\
\text { decay } \\
\text { mode }\end{array}$ & $\begin{array}{c}\text { Energy } \\
\text { difference } \\
\text { resolution } \\
(\mathrm{MeV})\end{array}$ & $\begin{array}{r}\psi / D \text { mass } \\
\text { resolution } \\
\left(\mathrm{MeV} / c^{2}\right) \\
\end{array}$ & Efficiency $^{c}$ & $\begin{array}{c}\text { Number of } \\
\text { events }^{d}\end{array}$ & $\begin{array}{l}\text { Branching } \\
\text { ratio }(\%)\end{array}$ \\
\hline$B^{-} \rightarrow D^{0} \pi^{-}$ & $\begin{array}{l}D^{0} \rightarrow K^{-} \pi^{+} \\
D^{0} \rightarrow K^{-} \pi^{+} \pi^{+} \pi^{-} \\
D^{0} \rightarrow \bar{K}^{0} \pi^{+} \pi^{-}\end{array}$ & $\begin{array}{l}21 \\
25\end{array}$ & $\begin{array}{r}11 \\
8 \\
9\end{array}$ & $\begin{array}{l}0.42 \\
0.27 \\
0.05\end{array}$ & $\begin{array}{l}19 \pm 5 \\
25 \pm 6 \\
10 \pm 4\end{array}$ & $\begin{array}{c}0.46 \pm 0.11 \\
0.43 \pm 0.09 \\
1.2 \pm 0.5\end{array}$ \\
\hline$D^{* 0} \pi^{-}$ & $\begin{array}{l}D^{* 0} \rightarrow D^{0} \gamma \\
D^{0} \rightarrow K^{-} \pi^{+} \\
D^{0} \rightarrow K^{-} \pi^{+} \pi^{+} \pi^{-}\end{array}$ & $\begin{array}{l}45 \\
45\end{array}$ & $\begin{array}{r}30 \\
11 \\
8\end{array}$ & $\begin{array}{l}0.13 \\
0.08\end{array}$ & $\begin{array}{r}9 \pm 3 \\
12 \pm 4\end{array}$ & $\begin{array}{l}0.73 \pm 0.26 \\
0.67 \pm 0.25\end{array}$ \\
\hline$D^{*+} \pi^{-} \pi^{-}$ & $\begin{array}{l}D^{*+} \rightarrow D^{0} \pi^{+} \\
D^{0} \rightarrow K^{-} \pi^{+} \\
D^{0} \rightarrow K^{-} \pi^{+} \pi^{+} \pi^{-}\end{array}$ & $\begin{array}{l}18 \\
20\end{array}$ & $\begin{array}{c}0.7 \\
11 \\
8\end{array}$ & $\begin{array}{l}0.23 \\
0.11\end{array}$ & $\begin{array}{l}<8 \\
<3.5\end{array}$ & $\begin{array}{l}<0.6 \\
<0.25\end{array}$ \\
\hline$D_{J}^{* * 0} \pi^{-}$ & $\begin{array}{l}D_{J}^{* * 0} \rightarrow D^{*+} \pi^{-} \\
D^{*+} \rightarrow D^{0} \pi^{+} \\
D^{0} \rightarrow K^{-} \pi^{+} \\
D^{0} \rightarrow K^{-} \pi^{+} \pi^{+} \pi^{-}\end{array}$ & $\begin{array}{l}20 \\
20\end{array}$ & $\begin{array}{c}\text { See text } \\
0.7 \\
11 \\
8\end{array}$ & $\begin{array}{l}0.22 \\
0.13\end{array}$ & $\begin{array}{l}2.2 \pm 1.5 \\
1.8 \pm 1.5\end{array}$ & $\begin{array}{l}0.17 \pm 0.11 \\
0.12 \pm 0.09\end{array}$ \\
\hline$D^{+} \pi^{-} \pi^{-}$ & $\begin{array}{l}D^{+} \rightarrow \bar{K}^{0} \pi^{+} \\
D^{+} \rightarrow K^{-} \pi^{+} \pi^{+}\end{array}$ & $\begin{array}{l}21 \\
17\end{array}$ & $\begin{array}{l}10 \\
10\end{array}$ & $\begin{array}{l}0.08 \\
0.27\end{array}$ & $\begin{array}{l}<5 \\
<16\end{array}$ & $\begin{array}{l}<0.8 \\
<0.3\end{array}$ \\
\hline$D_{1}^{* * 0} \pi^{-}$ & $\begin{array}{l}D_{1}^{* * 0} \rightarrow D^{+} \pi^{-} \\
D^{+} \rightarrow \bar{K}^{0} \pi^{+} \\
D^{+} \rightarrow K^{-} \pi^{+} \pi^{+}\end{array}$ & $\begin{array}{l}25 \\
20\end{array}$ & $\begin{array}{l}30 \\
10 \\
10\end{array}$ & $\begin{array}{l}0.06 \\
0.20\end{array}$ & $\begin{array}{l}<3 \\
<6\end{array}$ & $\begin{array}{l}<0.6 \\
<0.2\end{array}$ \\
\hline$D_{2}^{* * 0} \pi^{-}$ & $\begin{array}{l}D_{2}^{* * 0} \rightarrow D^{+} \pi^{-} \\
D^{+} \rightarrow \bar{K}^{0} \pi^{+} \\
D^{+} \rightarrow K^{-} \pi^{+} \pi^{+}\end{array}$ & $\begin{array}{l}25 \\
20\end{array}$ & $\begin{array}{l}30 \\
10 \\
10\end{array}$ & $\begin{array}{l}0.05 \\
0.17\end{array}$ & $\begin{array}{l}<2.3 \\
<2.6\end{array}$ & $\begin{array}{l}<0.6 \\
<0.07\end{array}$ \\
\hline$D^{0} \pi^{+} \pi^{-} \pi^{-}$ & $D^{0} \rightarrow K^{-} \pi^{+}$ & 17 & 11 & 0.32 & $34 \pm 8$ & $1.1 \pm 0.3$ \\
\hline$\psi K^{-}$ & $\psi \rightarrow \mu^{+} \mu^{-}$or $e^{+} e^{-}$ & 20 & 25 & 0.41 & $11 \pm 3$ & $0.08 \pm 0.02$ \\
\hline$\psi K^{*-}$ & $\psi \rightarrow \mu^{+} \mu^{-}$or $e^{+} e^{-}$ & 20 & 25 & 0.047 & $2 \pm 1$ & $0.13 \pm 0.09$ \\
\hline$\psi K^{-} \pi^{+} \pi^{-}$ & $\psi \rightarrow \mu^{+} \mu^{-}$or $e^{+} e^{-}$ & 20 & 25 & 0.14 & $6 \pm 3$ & $0.12 \pm 0.06$ \\
\hline$\psi^{\prime} K^{-}$ & $\begin{array}{l}\psi^{\prime} \rightarrow \mu^{+} \mu^{-} \text {or } e^{+} e^{-} \\
\psi^{\prime} \rightarrow \psi \pi^{+} \pi^{-} \\
\qquad \mu^{+} \mu^{-} \text {or } e^{+} e^{-}\end{array}$ & $\begin{array}{l}20 \\
20\end{array}$ & $\begin{array}{r}26 \\
3 \\
25\end{array}$ & $\begin{array}{l}0.53 \\
0.23\end{array}$ & $\begin{array}{l}<2.3 \\
<2.3\end{array}$ & $\begin{array}{l}<0.1 \\
<0.09\end{array}$ \\
\hline$\psi^{\prime} K^{*-}$ & $\begin{array}{l}\psi^{\prime} \rightarrow \mu^{+} \mu^{-} \text {or } e^{+} e^{-} \\
\psi^{\prime} \rightarrow \psi \pi^{+} \pi^{-} \\
\quad \stackrel{4}{\rightarrow} \mu^{+} \mu^{-} \text {or } e^{+} e^{-}\end{array}$ & $\begin{array}{l}15 \\
15\end{array}$ & $\begin{array}{r}26 \\
3 \\
25\end{array}$ & $\begin{array}{l}0.075 \\
0.03\end{array}$ & $\begin{array}{l}<2.3 \\
<2.3\end{array}$ & $\begin{array}{l}<0.7 \\
<0.7\end{array}$ \\
\hline $\bar{B}^{0} \rightarrow D^{+} \pi^{-}$ & $\begin{array}{l}D^{+} \rightarrow \bar{K}^{0} \pi^{+} \\
D^{+} \rightarrow K^{-} \pi^{+} \pi^{+}\end{array}$ & $\begin{array}{l}26 \\
22\end{array}$ & $\begin{array}{l}10 \\
10\end{array}$ & $\begin{array}{l}0.09 \\
0.33\end{array}$ & $\begin{array}{l}4 \pm 2 \\
17 \pm 4\end{array}$ & $\begin{array}{l}0.56 \pm 0.30 \\
0.23 \pm 0.06\end{array}$ \\
\hline$D^{*+} \pi^{-}$ & $\begin{array}{l}D^{*+} \rightarrow D^{0} \pi^{+} \\
D^{0} \rightarrow K^{-} \pi^{+} \\
D^{0} \rightarrow K^{-} \pi^{+} \pi^{+} \pi^{-}\end{array}$ & $\begin{array}{l}27 \\
24\end{array}$ & $\begin{array}{c}0.7 \\
11 \\
8\end{array}$ & $\begin{array}{l}0.34 \\
0.19\end{array}$ & $\begin{array}{l}8 \pm 3 \\
9 \pm 3\end{array}$ & $\begin{array}{l}0.40 \pm 0.15 \\
0.40 \pm 0.13\end{array}$ \\
\hline$D^{*+} \rho^{-}$ & $\begin{array}{l}D^{*+} \rightarrow D^{0} \pi^{+} \\
D^{0} \rightarrow K^{-} \pi^{+} \\
D^{0} \rightarrow K^{-} \pi^{+} \pi^{+} \pi^{-}\end{array}$ & $\begin{array}{l}125 \\
125\end{array}$ & $\begin{array}{c}0.7 \\
11 \\
8\end{array}$ & $\begin{array}{l}0.024 \\
0.016\end{array}$ & $\begin{array}{l}2 \pm 1 \\
4 \pm 2\end{array}$ & $\begin{array}{l}1.5 \pm 1.0 \\
2.0 \pm 1.0\end{array}$ \\
\hline$D^{0} \pi^{+} \pi^{-}$ & $\begin{array}{l}D^{0} \rightarrow K^{-} \pi^{+} \\
D^{0} \rightarrow K^{-} \pi^{+} \pi^{+} \pi^{-}\end{array}$ & $\begin{array}{l}19 \\
19\end{array}$ & $\begin{array}{r}11 \\
8\end{array}$ & $\begin{array}{l}0.34 \\
0.19\end{array}$ & $\begin{array}{l}<12 \\
<18\end{array}$ & $\begin{array}{l}<0.4 \\
<0.4\end{array}$ \\
\hline
\end{tabular}


TABLE IV. (Continued).

\begin{tabular}{|c|c|c|c|c|c|c|}
\hline $\begin{array}{l}B \text { decay } \\
\text { mode }^{\mathrm{a}}\end{array}$ & $\begin{array}{l}\text { Charm } \\
\text { decay } \\
\text { mode }\end{array}$ & $\begin{array}{c}\text { Energy } \\
\text { difference } \\
\text { resolution } \\
(\mathrm{MeV})\end{array}$ & $\begin{array}{r}\psi / D \text { mass } \\
\text { resolution } \\
\left(\mathrm{MeV} / \mathrm{c}^{\mathrm{b}}\right)\end{array}$ & Efficiency $^{c}$ & $\begin{array}{c}\text { Number of } \\
\text { events }\end{array}$ & $\begin{array}{l}\text { Branching } \\
\text { ratio }(\%)\end{array}$ \\
\hline $\bar{B}^{0} \rightarrow D_{0}^{* *+} \pi^{-}$ & $\begin{array}{l}D_{0}^{* *+} \rightarrow D^{0} \pi^{+} \\
D^{0} \rightarrow K^{-} \pi^{+} \\
D^{0} \rightarrow K^{-} \pi^{+} \pi^{+} \pi^{-}\end{array}$ & $\begin{array}{l}20 \\
20\end{array}$ & $\begin{array}{r}30 \\
11 \\
8\end{array}$ & $\begin{array}{l}0.25 \\
0.14\end{array}$ & $\begin{array}{l}<0.9 \\
<3\end{array}$ & $\begin{array}{l}<0.04 \\
<0.01\end{array}$ \\
\hline$D_{2}^{* *+} \pi^{-}$ & $\begin{array}{l}D_{2}^{* *+} \rightarrow D^{0} \pi^{+} \\
D^{0} \rightarrow K^{-} \pi^{+} \\
D^{0} \rightarrow K^{-} \pi^{+} \pi^{+} \pi^{-}\end{array}$ & $\begin{array}{l}19 \\
21\end{array}$ & $\begin{array}{r}30 \\
11 \\
8\end{array}$ & $\begin{array}{l}0.22 \\
0.13\end{array}$ & $\begin{array}{l}<4 \\
<7\end{array}$ & $\begin{array}{l}<0.2 \\
<0.3\end{array}$ \\
\hline$D^{0} \rho^{0}$ & $\begin{array}{l}D^{0} \rightarrow K^{-} \pi^{+} \\
D^{0} \rightarrow K^{-} \pi^{+} \pi^{+} \pi^{-}\end{array}$ & $\begin{array}{l}19 \\
18\end{array}$ & $\begin{array}{r}11 \\
8\end{array}$ & $\begin{array}{l}0.17 \\
0.11\end{array}$ & $\begin{array}{l}<1.7 \\
<3.4\end{array}$ & $\begin{array}{l}<0.10 \\
<0.14\end{array}$ \\
\hline$D^{*+} \pi^{-} \pi^{+} \pi^{-}$ & $\begin{array}{l}D^{*+} \rightarrow D^{0} \pi^{+} \\
D^{0} \rightarrow K^{-} \pi^{+} \\
D^{0} \rightarrow K^{-} \pi^{+} \pi^{+} \pi^{-}\end{array}$ & $\begin{array}{l}17 \\
16\end{array}$ & $\begin{array}{c}0.7 \\
11 \\
8\end{array}$ & $\begin{array}{l}0.15 \\
0.08\end{array}$ & $\begin{array}{l}18 \pm 4 \\
18 \pm 5\end{array}$ & $\begin{array}{l}2.1 \pm 0.6 \\
1.9 \pm 0.6\end{array}$ \\
\hline$D^{+} \pi^{-} \pi^{+} \pi^{-}$ & $\begin{array}{l}D^{+} \rightarrow \bar{K}^{0} \pi^{+} \\
D^{+} \rightarrow K^{-} \pi^{+} \pi^{+}\end{array}$ & $\begin{array}{l}19 \\
17\end{array}$ & $\begin{array}{l}10 \\
10\end{array}$ & $\begin{array}{l}0.06 \\
0.22\end{array}$ & $\begin{array}{l}11 \pm 4 \\
27 \pm 9\end{array}$ & $\begin{array}{c}2.7 \pm 1.0 \\
0.40 \pm 0.19\end{array}$ \\
\hline$\psi \bar{K}^{0}$ & $\psi \rightarrow \mu^{+} \mu^{-}$or $e^{+} e^{-}$ & 20 & 25 & 0.15 & $3 \pm 2$ & $0.06 \pm 0.03$ \\
\hline$\psi \bar{K}^{* 0}$ & $\psi \rightarrow \mu^{+} \mu^{-}$or $e^{+} e^{-}$ & 15 & 25 & 0.21 & $7 \pm 3$ & $0.11 \pm 0.05$ \\
\hline$\psi K^{-} \pi^{+}$ & $\psi \rightarrow \mu^{+} \mu^{-}$or $e^{+} e^{-}$ & 20 & 25 & 0.19 & $7 \pm 3$ & $0.10 \pm 0.04$ \\
\hline$\psi^{\prime} \overline{\boldsymbol{K}}^{0}$ & $\begin{aligned} \psi^{\prime} \rightarrow \mu^{+} \mu^{-} \text {or } e^{+} e^{-} \\
\psi^{\prime} \rightarrow \psi \pi^{+} \pi^{-} \\
\stackrel{\llcorner}{\longrightarrow} \mu^{+} \mu^{-} \text {or } e^{+} e^{-}\end{aligned}$ & $\begin{array}{l}15 \\
15\end{array}$ & $\begin{array}{r}26 \\
3 \\
25\end{array}$ & $\begin{array}{l}0.18 \\
0.07\end{array}$ & $\begin{array}{l}<2.3 \\
<2.3\end{array}$ & $\begin{array}{l}<0.3 \\
<0.3\end{array}$ \\
\hline$\psi^{\prime} \bar{K}^{* 0}$ & $\begin{array}{l}\psi^{\prime} \rightarrow \mu^{+} \mu^{-} \text {or } e^{+} e^{-} \\
\psi^{\prime} \rightarrow \psi \pi^{+} \pi^{-} \\
\stackrel{\leftrightarrow \mu^{+} \mu^{-}}{ } \text {or } e^{+} e^{-}\end{array}$ & $\begin{array}{l}15 \\
15\end{array}$ & $\begin{array}{r}26 \\
3 \\
25\end{array}$ & $\begin{array}{l}0.25 \\
0.10\end{array}$ & $\begin{array}{l}2 \pm 1 \\
1 \pm 1\end{array}$ & $\begin{array}{l}0.19 \pm 0.13 \\
0.09 \pm 0.09\end{array}$ \\
\hline
\end{tabular}

${ }^{\text {a The }} D^{* *}$ decay modes are subsets of the previously listed $D \pi \pi$ or $D^{*} \pi \pi$ mode.

${ }^{\mathrm{b}}$ For $D^{* *}$ decays, FWHM is listed.

${ }^{\text {c } T h i s ~ i n c l u d e s ~ d e t e c t o r ~ a n d ~ i d e n t i f i c a t i o n ~ e f f i c i e n c y . ~ F o r ~ t h e ~} \psi$ and $\psi^{\prime}$ modes, branching ratios of $K$ and $K^{*}$ to observable final states are included.

${ }^{\mathrm{d}}$ Limits are at $90 \%$ confidence level.

a good estimate of the background in the former. The background arises from three main sources: continuum events, fake charmed meson candidates, and combinations of real charmed mesons with some tracks from the other $B$. Figure 7 shows the measured $\Delta E$ distribution for $\bar{B} \rightarrow D \pi$ and $\bar{B} \rightarrow D^{*} \pi$.

Other selection criteria are applied to reduce the remaining background from both continuum and $B \bar{B}$ sources.

(i) Because the $B$ has spin 0, the distribution in the angle $\theta_{B}$ between the $B$ direction and the beam axis is proportional to $\sin ^{2} \theta_{B}$ whereas background events are distributed isotropically. We require $\left|\cos \theta_{B}\right|<0.8$.

(ii) In order to reduce background from two-jet continuum events, the event shape is examined. Because the $B$ 's are produced nearly at rest, the decay products of the two $B$ 's have very little angular correlation. Therefore, the distribution of the angle $\theta_{S}$ between the sphericity axes calculated from charged tracks forming the $B$ candidate and from those in the rest of the event should be isotropic for real $B$ candidates. The continuum background events fall mostly near $\cos \theta_{S}= \pm 1$. We require $\left|\cos \theta_{S}\right|<0.8$. The net effect of these two cuts is a $75 \%$ reduction in background with a loss of only $25 \%$ of the signal. The $\theta_{B}$ and $\theta_{S}$ cuts are not used in two-body modes that contain a $D^{*+}$ or $D^{+} \rightarrow K_{S}^{0} \pi^{+}$.

(iii) When a spin-0 particle decays into spin-1 and spin-0 particles, such as $B \rightarrow \psi K$ or $D^{*} \pi$, the helicity of the spin-1 particle is 0 . If one defines $\theta_{H}$ to be the angle between the vector-particle momentum and the direction of one of its decay products in the vector-particle rest frame, the decay rate is proportional to $\cos ^{2} \theta_{H}$ if the daughters have spin 0 , and $\sin ^{2} \theta_{H}$ if the vector-particle decays electromagnetically into lepton-antilepton pairs. For the $\psi K$ cases, we require $\left|\cos \theta_{H}\right|<0.85$. For $D^{0} \rho^{0}$ we require $\left|\cos \theta_{H}\right|>0.5$. 


\section{MASSES AND BRANCHING RATIOS}

\section{A. Masses of $B^{-}$and $\bar{B}^{0}$}

Figures 8 and 9 show the $B$ mass distributions for the $\Delta E$ signal (a) and sideband (b) regions for several modes that contain only charged particles and have low backgrounds. The candidates labeled $D^{*+}$ " $a_{1}$ " are a subset of the $D^{*+} \pi^{+} \pi^{-} \pi^{-}$candidates in which the three-pion invariant mass is required to be consistent within \pm 400 $\mathrm{MeV} / c^{2}$ of the $a_{1}$ mass of $1260 \mathrm{MeV} / c^{2}$. To obtain the $\bar{B}^{0}$ and $B^{-}$meson masses, we fit these distributions with a Gaussian signal with the expected rms resolution of 2.5 $\mathrm{MeV} / c^{2}$ and a background function, $w_{1} p+w_{2} p^{2}$ where $p=\sqrt{E_{\text {beam }}^{2}-m^{2}}$. The background function in the $\Delta E$ signal region (a) is constrained by simultaneously fitting the function to the mass plot from the $\Delta E$ sideband region (b).

We estimate the effect of initial-state radiation on the mass determination using the Jackson-Scharre formalism [32]. We use a Breit-Wigner cross-section function and require that after radiation the total cross section be maximized at the beam energy used in the $\Upsilon(4 S)$ data taking, the condition used to choose the energy. Because the form of the $\Upsilon(4 S)$ wave function is unknown, we have used phase-space factors in the Breit-Wigner function with a $p^{2}$ momentum dependence and use the results from the calculation with $p$ and $p^{3}$ momentum dependence to estimate the systematic error. We find the mean center-of-mass energy after radiation and correct the $B$ masses. We find this correction to be $-1.1 \pm 0.5$ $\mathrm{MeV} / \mathrm{c}^{2}$.

We find masses of the $B$ mesons to be

$$
\begin{aligned}
& m_{0}=5278.0 \pm 0.4 \pm 2.0 \mathrm{MeV} / c^{2}, \\
& m_{ \pm}=5278.3 \pm 0.4 \pm 2.0 \mathrm{MeV} / c^{2} .
\end{aligned}
$$

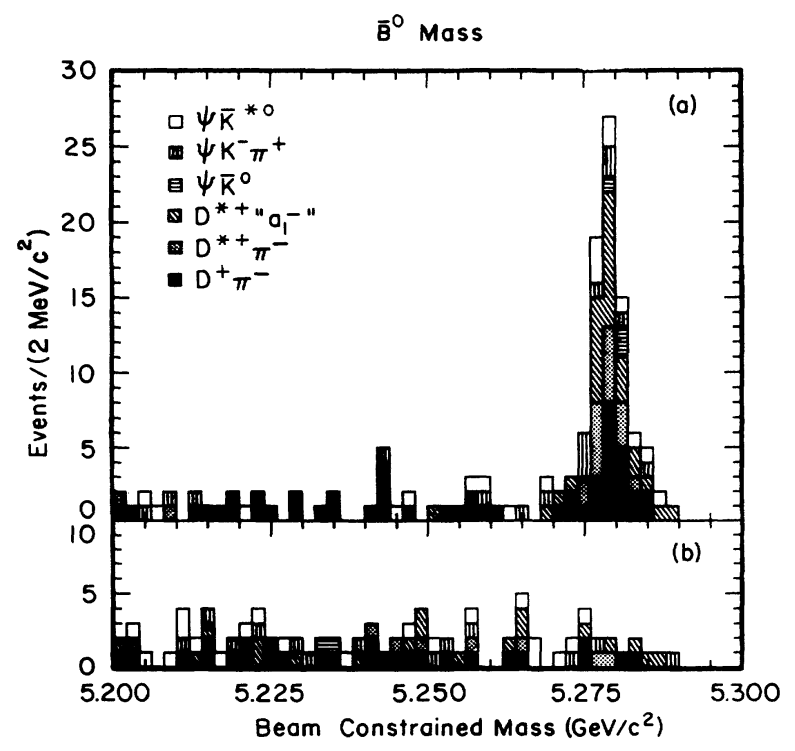

FIG. 8. Beam-constrained mass of reconstructed $\bar{B}^{0}$ candidates in modes used in the mass fit: (a) $|\Delta E|<2 \sigma_{E}$; (b) $2 \sigma_{E}<|\Delta E|<4 \sigma_{E}$.

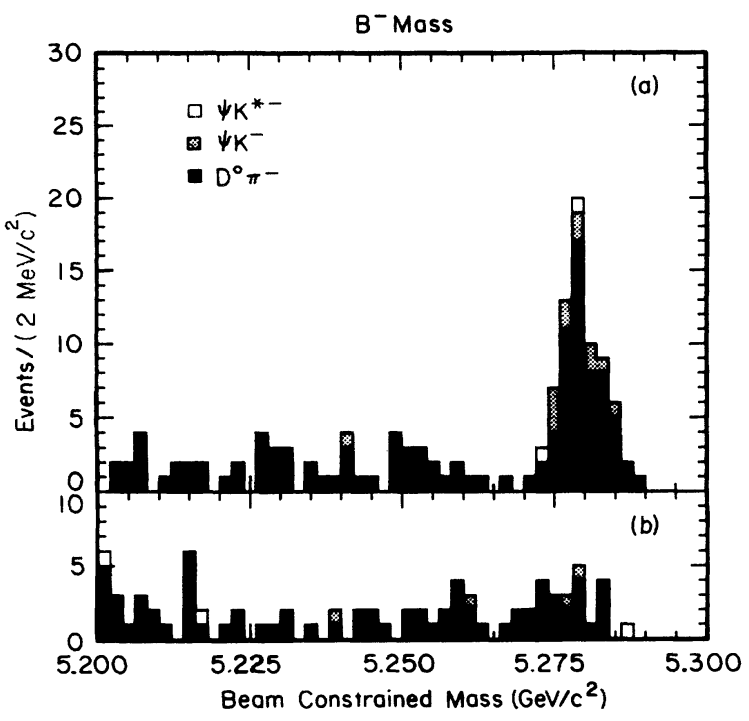

FIG. 9. Beam-constrained mass of reconstructed $B^{-}$candidates in modes used in the mass fit: (a) $|\Delta E|<2 \sigma_{E}$; (b) $2 \sigma_{E}<|\Delta E|<4 \sigma_{E}$.

The dominant systematic error in these measurements is due to the uncertainty in the CESR beam energy. The difference between the two masses is

$$
m_{0}-m_{ \pm}=-0.4 \pm 0.6 \pm 0.5 \mathrm{MeV} / c^{2} .
$$

The systematic error resulting from the uncertainty in $E_{\text {beam }}$ cancels in the difference. The remaining systematic error is estimated by choosing different background functions and by varying the criteria used to select events. This mass difference is in agreement with the ARGUS [33] measurement of $-0.9 \pm 1.2 \pm 0.5 \mathrm{MeV} / \mathrm{c}^{2}$ and marginally consistent with our previous measurement [31] of $2.0 \pm 1.1 \mathrm{MeV} / \mathrm{c}^{2}$. This mass difference is inconsistent with several theoretical predictions [34-37] which range from 1.2 to $2.3 \mathrm{MeV} / \mathrm{c}^{2}$.

\section{B. Branching ratios in two-body modes}

We obtain the number of $B$ decays in each mode by fitting the mass distribution of $B$ candidates with the background determined as described above and a Gaussian at the above-measured mass and with an rms width of $2.5 \mathrm{MeV} / c^{2}$. The efficiency for detecting a particular decay mode is calculated using a Monte Carlo simulation of the CLEO detector. These efficiencies are listed in Table IV. To obtain branching ratios, we correct the number of observed events with the detection efficiency and relevant intermediate particle branching ratios [15].

Where signals are observed, results from different charmed meson decay modes are averaged by dividing the total number of events observed by the sum of the products of the efficiencies and charm branching ratios in each submode. Where no significant signal is observed, we obtain upper limits by averaging results in each submode weighted by the product of efficiency and charm 
branching ratio. The quoted branching ratios $b_{ \pm}\left(b_{0}\right)$ for $B^{-}\left(\bar{B}^{0}\right)$ decay modes may differ from the true values $\widetilde{b}_{ \pm}$ $\left(\widetilde{b}_{0}\right)$ where $b_{ \pm}=2 f_{ \pm} \widetilde{b}_{ \pm}\left(b_{0}=2 f_{0} \widetilde{b}_{0}\right)$. Table $\mathrm{V}$ presents branching ratios in two-body modes containing a $D, D^{*}$, $\psi$, or $\psi^{\prime}$, combining results from the different charm modes listed in Table IV. Errors on the new measurements are, from left to right, statistical and systematic and those arising from uncertainty in the charmed particle branching ratio(s). The systematic error includes uncertainties in the efficiencies and fitting procedures. We also include results for multibody $\psi$ decay modes and for decays to a $D$ and a $D_{S}$ reported in Ref. [26] which have been recalculated to account for the average of recent measurements by the CLEO [23] and ARGUS [24] Collaborations of $\mathcal{B}\left(D_{S}^{+} \rightarrow \phi \pi^{+}\right)=(3.0 \pm 1.1) \%$. The table also includes measurements previously reported by the CLEO [31] and ARGUS [33] Collaborations and the predictions of the model of Bauer, Stech, and Wirbel [1]. The first and second errors in these other measurements are statistical and systematic, respectively. The errors from the charm branching ratios are of the same order as our systematic errors, except in modes which contain a photon or a neutral pion, in which case the efficiency error dominates. Therefore, the systematic error in the three sets of measurements are largely correlated. We prefer not to combine the present and earlier CLEO measurements, since they were obtained with different tracking chamber systems.

For most of the modes the measured branching fractions are consistent with the previous measurements by

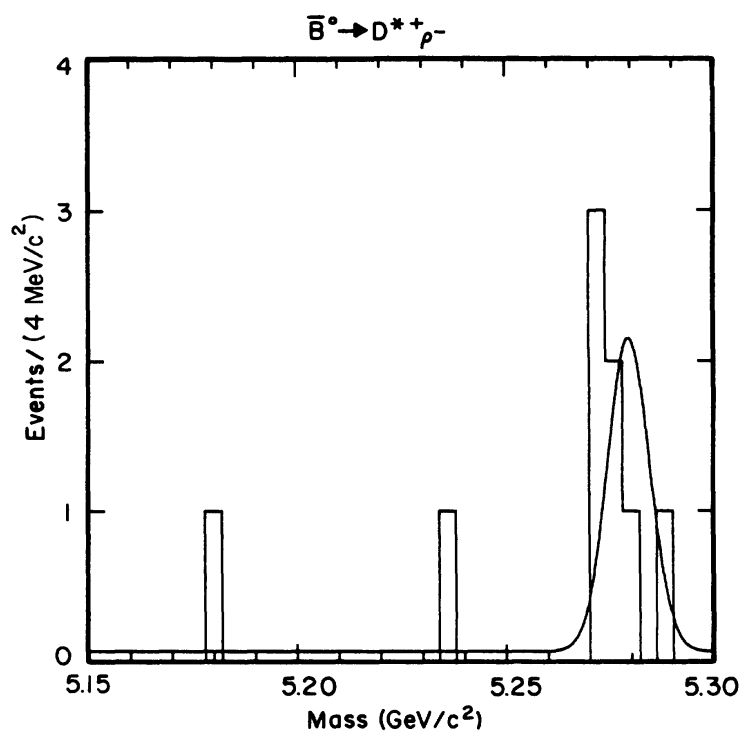

FIG. 10. Distribution in beam-constrained mass for $\bar{B}^{0} \rightarrow D^{*+} \rho^{-}$candidates.

the CLEO Collaboration and measurements by the ARGUS Collaboration. However, the measurements disagree by about two standard deviations for $D^{0} \pi^{-}$. We do not confirm the decay $B^{-} \rightarrow \psi^{\prime} K^{-}$reported by the

TABLE V. $B$ branching ratios (\%).

\begin{tabular}{|c|c|c|c|c|}
\hline Mode & $\begin{array}{c}\text { CLEO } \\
1987^{\mathrm{a}} \\
\end{array}$ & $\begin{array}{c}\text { CLEO } \\
1985[31]^{\mathrm{b}}\end{array}$ & ARGUS [33] & $\begin{array}{l}\text { Bauer et al. } \\
\text { model }[1]^{\mathrm{c}}\end{array}$ \\
\hline$B^{-} \rightarrow D^{0} \pi^{-}$ & $0.50 \pm 0.07 \pm 0.04 \pm 0.05$ & $0.55 \pm 0.17 \pm 0.11$ & $0.20 \pm 0.08 \pm 0.06$ & $0.48\left(a_{1}+0.75 a_{2}\right)^{2}$ \\
\hline$B^{-} \rightarrow D^{* 0} \pi^{-}$ & $0.72 \pm 0.18 \pm 0.14 \pm 0.07$ & & $0.40 \pm 0.14 \pm 0.12$ & $0.37\left(a_{1}+1.04 a_{2}\right)^{2}$ \\
\hline$B^{-} \rightarrow D^{*+} \pi^{-} \pi^{-}$ & $<0.4$ & $0.24 \pm 0.16 \pm 0.07$ & $0.26 \pm 0.14 \pm 0.07$ & \\
\hline$B^{-} \rightarrow \psi K^{-}$ & $0.08 \pm 0.02 \pm 0.02 \pm 0.01$ & $0.10 \pm 0.07 \pm 0.2$ & $0.07 \pm 0.03 \pm 0.01$ & $1.01 a_{2}^{2}$ \\
\hline$B^{-} \rightarrow \psi K^{*-}$ & $0.13 \pm 0.09 \pm 0.02 \pm 0.02$ & & $0.16 \pm 0.11 \pm 0.03$ & $4.33 a_{2}^{2}$ \\
\hline$B^{-} \rightarrow \psi K^{-} \pi^{+} \pi^{-}$ & $0.12 \pm 0.06 \pm 0.03 \pm 0.02$ & & $<0.16$ & \\
\hline$B^{-} \rightarrow \psi^{\prime} K^{-}$ & $<0.05$ & & $0.18 \pm 0.08 \pm 0.04$ & $0.28 a_{2}^{2}$ \\
\hline$B^{-} \rightarrow \psi^{\prime} K^{*-}$ & $<0.35$ & & $<0.49$ & $1.91 a_{2}^{2}$ \\
\hline$B^{-} \rightarrow D^{0} D_{S}^{-}$ & $1.9 \pm 0.8 \pm 0.2 \pm 0.7$ & & & $0.73 a_{1}^{2}$ \\
\hline$\overline{\boldsymbol{B}}^{0} \rightarrow D^{+} \pi^{-}$ & $0.27 \pm 0.06 \pm 0.03 \pm 0.04$ & $0.52 \pm 0.27 \pm 0.14$ & $0.48 \pm 0.11 \pm 0.11$ & $0.48 a_{1}^{2}$ \\
\hline $\bar{B}^{0} \rightarrow D^{*+} \pi^{-}$ & $0.40 \pm 0.10 \pm 0.04 \pm 0.06$ & $0.29 \pm 0.14 \pm 0.08$ & $0.28 \pm 0.09 \pm 0.06$ & $0.37 a_{1}^{2}$ \\
\hline $\bar{B}^{0} \rightarrow D^{*+} \rho^{-}$ & $1.9 \pm 0.8 \pm 1.1 \pm 0.3$ & & $0.7 \pm 0.3 \pm 0.3$ & $1.18 a_{1}^{2}$ \\
\hline $\bar{B}^{0} \rightarrow D^{*+} a_{1}^{-}$ & $1.8 \pm 0.6 \pm 0.5 \pm 0.3$ & & & $1.63 a_{1}^{2}$ \\
\hline $\bar{B}^{0} \rightarrow D^{0} \underline{\rho}^{0}$ & $<0.06$ & & & $0.07 a_{2}^{2}$ \\
\hline $\bar{B}^{0} \rightarrow \psi \bar{K}^{0}$ & $0.06 \pm 0.03 \pm 0.02 \pm 0.01$ & & $0.08 \pm 0.06 \pm 0.02$ & $1.02 a_{2}^{2}$ \\
\hline $\bar{B}^{0} \rightarrow \psi \bar{K}^{* 0}$ & $0.11 \pm 0.05 \pm 0.03 \pm 0.01$ & $0.35 \pm 0.16 \pm 0.03$ & $0.11 \pm 0.05 \pm 0.02$ & $4.36 a_{2}^{2}$ \\
\hline $\bar{B}^{0} \rightarrow \psi K^{-} \pi^{+}$ & $0.10 \pm 0.04 \pm 0.03 \pm 0.01$ & & $<0.10$ & \\
\hline $\bar{B}^{0} \rightarrow \psi^{\prime} \bar{K}^{0}$ & $<0.15$ & & $<0.28$ & $0.28 a_{2}^{2}$ \\
\hline $\bar{B}^{0} \rightarrow \psi^{\prime} \bar{K}^{* 0}$ & $0.14 \pm 0.08 \pm 0.03 \pm 0.02$ & & $<0.23$ & $1.91 a_{2}^{2}$ \\
\hline $\bar{B}^{0} \rightarrow D^{+} D_{S}^{-}$ & $0.80 \pm 0.45 \pm 0.06 \pm 0.29$ & & & $0.67 a_{1}^{2}$ \\
\hline $\bar{B}^{0} \rightarrow D^{*+} D_{S}^{-}$ & $1.6 \pm 0.9 \pm 0.2 \pm 0.6$ & & & $0.30 a_{1}^{2}$ \\
\hline
\end{tabular}

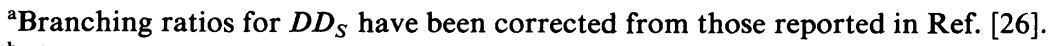

${ }^{b}$ The previous CLEO results have been renormalized for equal charged and neutral $B$ production on the $\Upsilon(4 S)$ and revised charm branching ratios.

${ }^{\mathrm{c}}$ Assumes $f_{D}=f_{D_{S}}=162 \mathrm{MeV}$ and $f_{D^{*}}=221 \mathrm{MeV}$. 
ARGUS Collaboration.

The $B$ mass plot for $\bar{B}^{0} \rightarrow D^{*+} \rho^{-}$is shown in Fig. 10 . Since the requirement on $\Delta E$ for $\bar{B}^{0} \rightarrow D^{*+} \rho^{-}$is not very stringent because of poor photon energy resolution of the CLEO detector, this mode might have background from other $B$ decays, such as $\bar{B}^{0} \rightarrow D^{*+} a_{1}^{-}$where one of the $a_{1}$ daughters has been missed. A Monte Carlo simulation shows, however, that the effect due to $\bar{B}^{0} \rightarrow D^{*+} a_{1}^{-}$is small since the measured branching fraction for $\bar{B}^{0} \rightarrow D^{*+} a_{1}^{-}$is comparable to that of $\bar{B}^{0} \rightarrow D^{*+} \rho^{-}$(see below). The large error in the branching ratio results from the dependence of the efficiency on the unknown $\rho$ polarization.

\section{Branching ratios for $\bar{B} \rightarrow D \pi \pi \pi$}

We have measured branching ratios for modes including a $D$ or a $D^{*}$ and three charged pions and have searched for resonant substructure in these decay modes. Table IV lists the observed yields and the efficiencies assuming the decay products are distributed as four-body phase space. The large combinatoric background precludes the possibility of observing a signal for $B^{-} \rightarrow D^{0} \pi^{+} \pi^{-} \pi^{-}, D^{0} \rightarrow K^{-} \pi^{+} \pi^{+} \pi^{-}$, so this submode is not considered. In the case of $D^{*}$, the efficiency is higher by a factor of 1.4 if the decay is actually $D^{*} a_{1}$.

Because the statistical significance of the $D \pi \pi \pi$ measurements is low, we confirm our results using an alternate technique. We require the $B$-candidate mass to be within $6 \mathrm{MeV} / \mathrm{c}^{2}$ of the measured value and plot the $\Delta E$ distributions. We fit these to a Gaussian signal with a mean of zero and a width determined by Monte Carlo simulations, plus a polynomial background. Figure 11 shows the mass and $\Delta E$ distributions for

(1) $\min \left(\left|\boldsymbol{M}_{\pi \pi, 1}-\boldsymbol{M}_{\rho}\right|,\left|\boldsymbol{M}_{\pi \pi, 2}-\boldsymbol{M}_{\rho}\right|\right)<0.115 \mathrm{GeV} / c^{2}$

(2) $\min \left(\left|M_{\pi \pi, 1}-M_{\rho}\right|,\left|M_{\pi \pi, 2}-M_{\rho}\right|\right)<0.115 \mathrm{GeV} / c^{2}$,

(3) $\min \left(\left|\boldsymbol{M}_{\pi \pi, 1}-\boldsymbol{M}_{\rho}\right|,\left|\boldsymbol{M}_{\pi \pi, 2}-\boldsymbol{M}_{\rho}\right|\right)>0.115 \mathrm{GeV} / c^{2}$

for the $\Delta E$ signal and sideband regions, where $M_{\pi \pi, 1}$ and $M_{\pi \pi, 2}$ are the masses of the two $\pi^{+} \pi^{-}$combinations. We find the net yield by subtracting the sideband measurements from those in the signal region and then calculate the fraction of events in each sample. We also calculate the fraction in each sample for Monte Carlo events generated in three decay modes: $D \pi \pi \pi, D \rho^{0} \pi$, and $D a_{1}$. We have used the $a_{1}$ mass and width as reported by Bowler [38] and assume that all its decays are in the $L=0$ partial wave. The efficiencies in each sample in each mode form a $3 \times 3$ matrix. The measured yields are multiplied by the inverse of that matrix to solve for the fraction of events from each process. Where two $D$ decay modes are used, we combine results with a $\chi^{2}$ fit, subject to the requirement that the sum of the fractions is 1 . To calculate the branching ratios, we multiply the efficiency-corrected total yield for each mode by the mea-
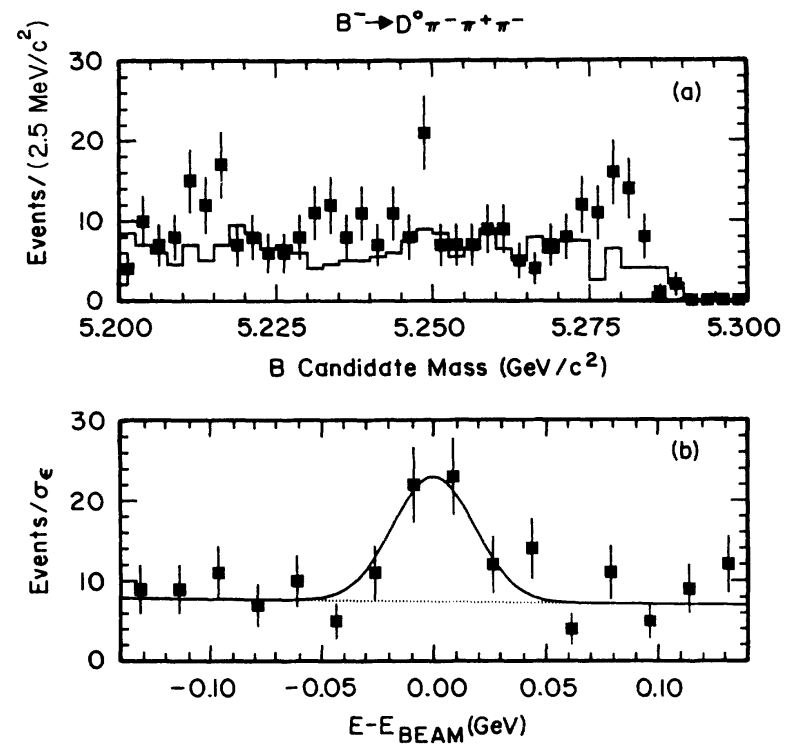

FIG. 11. (a) Distribution of beam-constrained mass for $B^{-} \rightarrow D^{0} \pi^{--} \pi^{+} \pi^{-}$candidates with $D^{0} \rightarrow K^{-} \pi^{+}$. The points show the yield from the $\Delta E$ signal region, and the histogram from the sideband region. (b) $\Delta E$ distribution for candidates within $6 \mathrm{GeV} / c^{2}$ of the measured $B$ mass. The curve shows the results of the fit.

$B^{-} \rightarrow D^{0} \pi^{-} \pi^{+} \pi^{-}, D^{0} \rightarrow K^{-} \pi^{+}$candidates.

To search for resonant substructure in these multibody $B$ decays we require that the $B$ mass be within $6 \mathrm{MeV} / c^{2}$ of the measured value and divide those data into three samples:

sured fractions. Table VI shows the measured fractions and net branching ratios. The errors shown are statistical and systematic. The systematic error is dominated by uncertainties sin the fitting procedures which are estimated by considering alternate background estimates. The measured branching ratios have been corrected for the unseen decay $a_{1}^{-} \rightarrow \rho^{-} \pi^{0}$ where appropriate. In $D^{*} a_{1}$ decays, the relative angular momentum can be 0,1 , or 2 . The systematic error in the branching ratios for $D^{*+} \pi^{+} \pi^{-} \pi^{-}$contains a contribution from the dependence of the efficiency on the unknown density of states.

To find the total $\bar{B} \rightarrow D \pi^{+} \pi^{-} \pi^{-}$branching ratios, we average the efficiencies according to the measured resonant fractions and include the uncertainty from this average in the systematic error. We find the inclusive fourbody rates to be $\mathscr{B}\left(B^{-} \rightarrow D^{0} \pi^{+} \pi^{-} \pi^{-}\right)=(1.15 \pm 0.29 \pm 0.13 \pm 0.16) \%$, 
TABLE VI. Four-body branching ratios (\%).

\begin{tabular}{|c|c|c|c|c|c|}
\hline & & $\begin{array}{c}D \pi^{+} \pi^{-} \pi^{-} \\
\text {(nonresonant) }\end{array}$ & $\begin{array}{c}D \rho^{0} \pi^{-} \\
\text {(nonresonant) }\end{array}$ & $D a_{1}^{-}$ & $\begin{array}{l}\text { Bauer et al. } \\
\text { model [1] for } D a_{1}^{-}\end{array}$ \\
\hline \multirow[t]{2}{*}{$D^{0}$} & Fraction & $0.48 \pm 0.30 \pm 0.20$ & $0.34 \pm 0.17 \pm 0.15$ & $0.18 \pm 0.06 \pm 0.12$ & \\
\hline & $\begin{array}{l}\text { Branching } \\
\text { ratio }(\%)\end{array}$ & $0.51 \pm 0.34 \pm 0.23$ & $0.42 \pm 0.23 \pm 0.20$ & $0.45 \pm 0.19 \pm 0.31$ & $1.25 a_{1}^{2}$ \\
\hline \multirow[t]{2}{*}{$D^{+}$} & Fraction & $0.50 \pm 0.13 \pm 0.15$ & $0.13 \pm 0.11 \pm 0.06$ & $0.37 \pm 0.10 \pm 0.14$ & \\
\hline & $\begin{array}{l}\text { Branching } \\
\text { ratio }(\%)\end{array}$ & $0.39 \pm 0.14 \pm 0.13$ & $0.11 \pm 0.09 \pm 0.04$ & $0.60 \pm 0.22 \pm 0.24$ & $1.25 a_{1}^{2}$ \\
\hline \multirow[t]{2}{*}{$D^{*+}$} & Fraction & $0.00 \pm 0.10 \pm 0.08$ & $0.35 \pm 0.15 \pm 0.08$ & $0.65 \pm 0.16 \pm 0.14$ & \\
\hline & $\begin{array}{l}\text { Branching } \\
\text { ratio }(\%)\end{array}$ & $0.00 \pm 0.19 \pm 0.16$ & $0.68 \pm 0.32 \pm 0.21$ & $1.82 \pm 0.55 \pm 0.55$ & $1.62 a_{1}^{2}$ \\
\hline
\end{tabular}

$\mathcal{B}\left(\bar{B}^{0} \rightarrow D^{+} \pi^{+} \pi^{-} \pi^{-}\right)=(0.80 \pm 0.21 \pm 0.09 \pm 0.11) \%$,

$\mathcal{B}\left(\bar{B}^{0} \rightarrow D^{*+} \pi^{+} \pi^{-} \pi^{-}\right)=(1.59 \pm 0.28 \pm 0.26 \pm 0.26) \%$ ，

where the errors are statistical, systematic, and that arising from charm branching ratios, respectively. Although the four-body decays are clearly observed, the results of Table VI show that with the available data it is not yet possible to measure the resonant substructure with precision. In the case of the $D^{0}$ and $D^{+}$, large backgrounds and low statistics are to blame, while in the case of $D^{*+}$, the number of events is insufficient to differentiate between the various angular momentum states. The observed total $D^{*+} \pi^{+} \pi^{-} \pi^{-}$rate is in good agreement with the ARGUS [33] measurement of $(1.2 \pm 0.3 \pm 0.4) \%$.

\section{Branching ratios for $\bar{B} \rightarrow D \pi \pi$}

We have measured branching ratios for modes including a $D$ or a $D^{*}$ and two charged pions and have searched for resonant substructure in these decay modes. We search for $\bar{B} \rightarrow D_{J}^{* *} \pi$ in $\bar{B} \rightarrow D \pi \pi$ and $\bar{B} \rightarrow D^{*} \pi \pi$ decays by requiring a $D \pi$ or $D^{*} \pi$ combination with a mass consistent with the expected $D^{* *}$ mass. For the $D_{1}^{* *}$ and masses and widths, $D_{2}^{* *}$ we use values measured by the CLEO Collaboration [39]. For the $D_{0}^{* *}$ mass and width, we use 2340 and $30 \mathrm{MeV} / \mathrm{c}^{2}$, as estimated by a CLEO study [40]. These values are not well determined, but are consistent with theoretical expectations [41]. In order to minimize the effect of the uncertainties in the $D^{* *}$ masses and widths on the efficiencies, we make the loose requirement that the $D_{J}^{* *}$ candidate mass to be within 50 $\mathrm{MeV} / c^{2}$ of the expected value. For $D^{*+} \pi^{-}$combinations, we require that either the $D_{1}^{* *} \pi$ or the $D_{2}^{* *} \pi$ hypothesis be satisfied. We then fit the $B$ mass distribution using the method outlined above. To suppress background observed in the $\Delta E$ sideband region and in continuum data, in the $D \pi \pi$ modes, we place a requirement on the angle $\left(\theta_{* *}\right)$ between the $D_{J}^{* *}$ candidate direction in the laboratory frame and the daughter pion in the $D \pi$ frame in addition to the cuts on $\theta_{B}$ and $\theta_{S}$ described earlier. We require $\cos \theta_{* *}>-0.7$. Product branching ratios are given in Table VII. No signal is observed for $\bar{B} \rightarrow D \pi \pi$. We observe 4 events consistent with $B \rightarrow D_{J}^{* * 0} \pi^{-}, D_{J}^{* * 0} \rightarrow D^{*+} \pi^{-}$. The branching ratio of $\left(0.14_{-0.06}^{+0.08} \pm 0.03\right) \%$ is in good agreement with the ARGUS measurement [33]. It is not possible to classify these events as either $D_{1}^{* *}$ or $D_{2}^{* *}$. The rightmost column in Table VII lists limits on the total $\bar{B} \rightarrow D \pi \pi$ branching fraction where we model the decays as phase space in the Monte Carlo efficiency calculation.

\section{E. Fit of parameters in exclusive $B$ decay model}

Bauer, Stech, and Wirbel $[1,43]$ (BSW) have predicted two-body $B$-decays branching ratios based on a factorization approach. The parameters $a_{1}$ and $a_{2}$ are proportional to the amplitudes for the effective charged current and effective neutral current decays. Tables V and VI present their predictions. Most modes can be described with only one of these numbers, but $D^{0} \pi^{-}$requires both. The $\psi$ can only be produced via the internal $W$ emission diagram and therefore its production in $B$ decay depends only on $a_{2}$. The branching ratios for $D D_{S}$ decay modes are proportional to the square of the $D_{S}$ decay constant $\left(f_{D_{S}}\right)$ which is unmeasured.

To determine the values of $a_{1}, a_{2}$, and $f_{D_{S}}$, we fit our measurements to the predictions of the BSW model. We include in our error matrix the correlations between

TABLE VII. Three-body branching ratios (\%). Limits are at $90 \%$ confidence level.

\begin{tabular}{lcccc}
\hline \hline & $\bar{B} \rightarrow D_{0}^{* *} \pi$ & $\bar{B} \rightarrow D_{2}^{* *} \pi$ & $\bar{B} \rightarrow D_{J}^{* *} \pi$ & $\bar{B} \rightarrow D \pi \pi$ \\
& $D_{0}^{* *} \rightarrow D \pi$ & $D_{2}^{* *} \rightarrow D \pi$ & $D_{J}^{* *} \rightarrow D^{*} \pi$ & Total \\
\hline$D^{+} \pi^{-} \pi^{-}$ & $<0.5$ & $<0.4$ & & $<0.7$ \\
$D^{0} \pi^{-} \pi^{+}$ & $<0.01$ & $<0.04$ & & $<0.7$ \\
$D^{*+} \pi^{-} \pi^{-}$ & & & $0.14_{-0.06}^{+0.08} \pm 0.01 \pm 0.02$ & $<0.4$ \\
\hline \hline
\end{tabular}


TABLE VIII. Results of fit for $B$ decay parameters.

\begin{tabular}{ccrrrc}
\hline \hline Fit & $a_{1}$ & \multicolumn{1}{c}{$a_{2}$} & $f_{D_{S}}$ & $\chi^{2} / N_{\text {DF }}$ & $\xi$ \\
\hline 1 & $0.85 \pm 0.06$ & $0.19 \pm 0.03$ & $226 \pm 78$ & $10.1 / 10$ & $0.45 \pm 0.03$ \\
2 & $0.92 \pm 0.07$ & $-0.17 \pm 0.03$ & $208 \pm 72$ & $17.8 / 10$ & $0.08 \pm 0.04$ \\
\hline \hline
\end{tabular}

charm branching ratios [44]. We assume that the $B$ lifetime is $\tau_{B}=1.2 \mathrm{ps}$, and the $b \rightarrow c$ CKM matrix element is $\left|V_{c b}\right|=0.05$. To compare to results using different values, our results for $a_{1}$ and $a_{2}$ should be scaled by $\left(\tau_{B} / 1.2 \mathrm{ps}\right)\left(\left|V_{c b}\right| / 0.05\right)^{2}$. The absolute values of $a_{1}$ and $a_{2}$ are well determined by the decay modes which are described by only one of the two. The relative sign is determined by the modes described by both. However, there are several large sources of uncertainty in determining the relative sign.

(a) For the modes described by both $a_{1}$ and $a_{2}$, the coefficient multiplying $a_{2}$ is proportional to the decay constant for $D$ or $D^{*}$. These decay constants are unmeasured, and there is large theoretical disagreement on their values [45].

(b) We have measured branching ratios in only two modes that can be used to determine the relative sign. The uncertainty in the measurements of the branching ratios in these modes is large relative to the uncertainty in the values of $a_{1}$ and $a_{2}$ determined independently.

(c) Variations in other quantities, such as charm branching ratios and the unmeasured form factors can also change the relative sign of $a_{1}$ and $a_{2}$. Therefore, we perform the fit for either relative sign between $a_{1}$ and $a_{2}$. The results are given in Table VIII along with the $\chi^{2}$ per degree of freedom of the fit. Because of the large uncer-

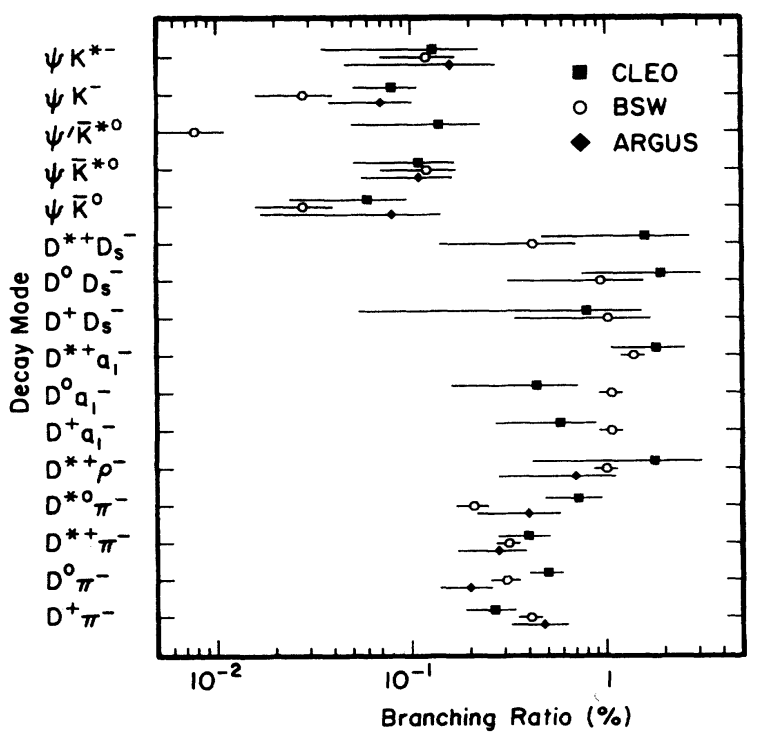

FIG. 12. CLEO and ARGUS measurements of various twobody decay modes and the predictions of the model of Bauer, Stech, and Wirbel using the fit to the factorization model parameters $a_{1}$ and $a_{2}$ derived from various $B$ decay modes. tainties in the determination of the sign, we do not favor the results of one fit over the other. Varying the correlation between the efficiencies used in deriving the measured branching ratios did not significantly alter the results.

QCD predicts values for the decay amplitude parameters [46] $c_{1}\left(m_{B}\right)=1.13$ and $c_{2}\left(m_{B}\right)=-0.29$, where

$$
\begin{aligned}
& a_{1}=c_{1}+\xi c_{2}, \\
& a_{2}=c_{2}+\xi c_{1}
\end{aligned}
$$

and $\xi$ is a free parameter that describes the level of color suppression. Table VIII also shows the value of $\xi$ derived from the value of $a_{1} / a_{2}$ in each of the fits. Figure 12 shows the measurements of the CLEO and ARGUS Collaborations and the predictions of the BSW model using the parameter values from Fit 2, since the theory and results from $D$ decays [1] favor the relative negative sign and a small level of color suppression.

\section{CONCLUSIONS}

We have measured the exclusive branching ratios of several decay modes. $\bar{B}^{0} \rightarrow D^{*+} a_{1}^{-}, D^{+} \pi^{+} \pi^{-} \pi^{-}$, and $B^{-} \rightarrow D^{0} \pi^{+} \pi^{-} \pi^{-}$have been observed for the first time. The mass difference between $\bar{B}^{0}$ and $B^{-}$has been measured to be $-0.4 \pm 0.6 \pm 0.5 \mathrm{MeV} / c^{2}$. The measured exclusive branching ratios are consistent with the factorization model. The measured inclusive $B \rightarrow D X$ momentum spectra agree well with factorization at high $D$ momentum; however, there is an excess in the data over the prediction at low momentum.

\section{ACKNOWLEDGMENTS}

We are grateful for the efforts of the entire CESR staff, which made this work possible. We also wish to thank M. Wirbel for helpful correspondence. The work was supported by the National Science Foundation and the U.S. Department of Energy under Contract Nos. DEAC02-76ER01428, DE-AC02-76ER03064, DE-AC0276ER01545, DE-AC02-78ER05001, DE-AC0283ER40105, and DE-FG05-86ER40272. The supercomputing resources of the Cornell Theory Center were used in this research. P.S.D. thanks the Presidential Young Investigator Program of the NSF and R.P. thanks the Sloan Foundation for their support. 
[1] M. Bauer, B. Stech, and M. Wirbel, Z. Phys. C 34, 103 (1987).

[2] D. Fakirov and B. Stech, Nucl. Phys. B133, 315 (1978).

[3] J. G. Korner, in Proceedings of the International Symposium on Production and Decay of Heavy Hadrons, Heidelberg, Germany, 1986, edited by K. R. Schubert and R. Waldi (DESY, Hamburg, 1987), p. 279.

[4] A. Ali et al., Z. Phys. C 1, 269 (1979).

[5] F. Hussain and M. Scadron, Phys. Rev. D 30, 1492 (1984).

[6] M. Wirbel and Y.-L. Wu, Phys. Lett. B 288, 430 (1989).

[7] D. Andrews et al., Nucl. Instrum. Methods 211, 47 (1983).

[8] D. Cassel et al., Nucl. Instrum. Methods A252, 325 (1986).

[9] S. Behrends et al., Phys. Rev. D 31, 2161 (1985).

[10] Modifications for the new drift chamber are described in CLEO internal memos CBX 87-52 and 87-53 (unpublished).

[11] G. Fox and S. Wolfram, Phys. Rev. Lett. 41, 1581 (1978).

[12] G. P. Lepage, Phys. Rev. D 42, 3251 (1990).

[13] One expects $\left(1-f_{B \bar{B}}\right)$ is of order $0.1 \%$, based on the relative widths of the three bound upsilon resonances and the $\Upsilon(4 S)$. The CLEO Collaboration previously reported [J. Alexander et al., Phys. Rev. Lett. 64, 2226 (1990)] evidence for non- $B \bar{B}$ decays of $\Upsilon(4 S)$ to high-momentum $\psi$ 's. However, in a more recent data set we find that the yield of high-momentum $\psi$ 's is consistent with continuum production.

[14] J. Alexander et al., Phys. Rev. Lett. 65, 1184 (1990).

[15] J. Adler et al., Phys. Rev. Lett. 60, 89 (1988).

[16] J. Adler et al., Phys. Lett. B 208, 153 (1988).

[17] Particle Data Group, J. J. Hernández et al., Phys. Lett. B 239, 1 (1990).

[18] D. Bortoletto et al., Phys. Rev. D 35, 19 (1988). Results have been rescaled to account for the branching ratios in Refs. [15] and [16].

[19] H. Albrecht et al., Report No. DESY-91-023 (unpublished). Branching ratios have been rescaled to account for the branching ratios in Refs. [15] and [16].

[20] M. Bauer, B. Stech, and M. Wirbel, Z. Phys. C 29, 637 (1985).

[21] H. Pietschmann and H. Rupertsberger, Z. Phys. C 27, 73
(1985).

[22] CLEO Collaboration, G. Crawford et al., Phys. Rev. D (to be published).

[23] J. Alexander et al., Phys. Rev. Lett. 65, 1531 (1990).

[24] H. Albrecht et al., Phys. Lett. B 255, 634 (1991).

[25] J. C. Anjos et al., Phys. Rev. Lett. 62, 722 (1990).

[26] D. Bortoletto et al., Phys. Rev. Lett. 64, 2117 (1990).

[27] B. Gittelman and S. Stone, in High Energy Electron Positron Physics, edited by A. Ali and P. Söding (World Scientific, Singapore, 1988), p. 275.

[28] S. Behrends et al., Phys. Rev. Lett. 59, 407 (1987).

[29] R. Kowalewski, Ph.D. dissertation, Cornell University, 1988.

[30] R. Fulton et al., Phys. Rev. D 43, 651 (1991).

[31] C. Bebek et al., Phys. Rev. D 36, 1289 (1987).

[32] J. D. Jackson and D. L. Scharre, Nucl. Instrum. Methods 128, 13 (1975).

[33] H. Albrecht et al., Z. Phys. C 48, 583 (1990).

[34] C. P. Singh et al., Phys. Rev. D 24, 788 (1981).

[35] L.-H. Chan, Phys. Rev. Lett. 51, 253 (1983).

[36] K. P. Tiwari et al., Phys. Rev. D 31, 642 (1985).

[37] D. Y. Kim and S. N. Sinha, Ann. Phys. (N.Y.) 42, 47 (1985).

[38] M. Bowler, Phys. Lett. B 209, 99 (1988).

[39] P. Avery et al., Phys. Rev. D 41, 774 (1990).

[40] H. Worden, CLEO internal memorandum CBX 89-54 (unpublished).

[41] J. L. Rosner, Comments Nucl. Part. Phys. 16, 109 (1986).

[42] S. Godfrey and N. Isgur, Phys. Rev. D 32, 189 (1985).

[43] Predictions for two-body $B \rightarrow \psi^{\prime}$ come from $\mathbf{M}$. Wirbel (private communication).

[44] The error matrix for the fit of $D$ branching ratios in Ref. [15] was provided by G. Gladding (private communication).

[45] J. Rosner, Phys. Rev. D 42, 3732 (1990), provides a summary of predictions for $f_{D}$ and $f_{D_{S}}$ which vary over ranges of 117 to $240 \mathrm{MeV}$ and 129 to $290 \mathrm{MeV}$, respectively. We follow BSW and assume $f_{D}=162 \mathrm{MeV}$ and $f_{D^{*}}=221 \mathrm{MeV}$.

[46] R. Rükl, Habilitationsschrift, University of Munich, 1983. 


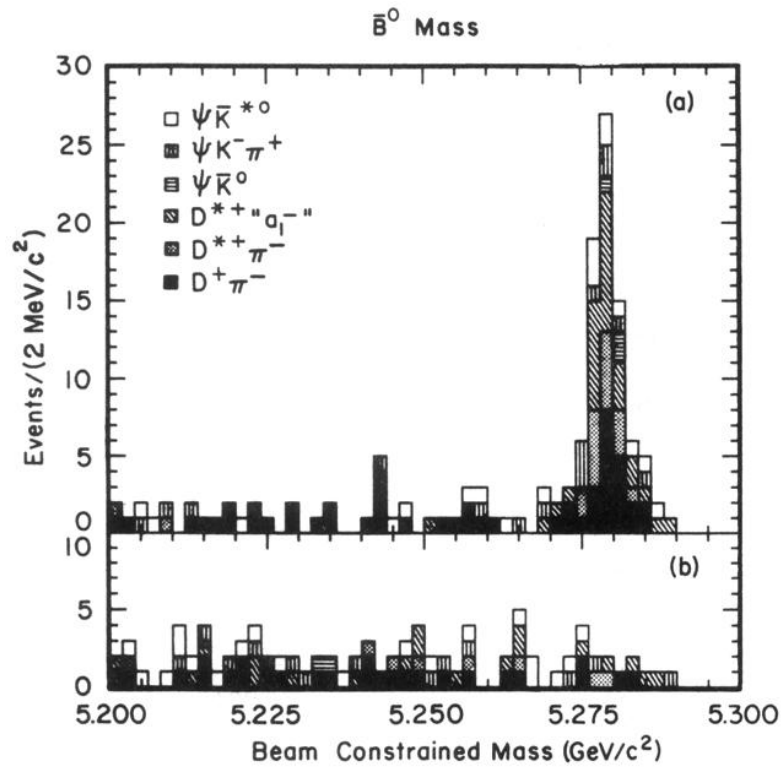

FIG. 8. Beam-constrained mass of reconstructed $\bar{B}^{0}$ candidates in modes used in the mass fit: (a) $|\Delta E|<2 \sigma_{E}$; (b) $2 \sigma_{E}<|\Delta E|<4 \sigma_{E}$. 


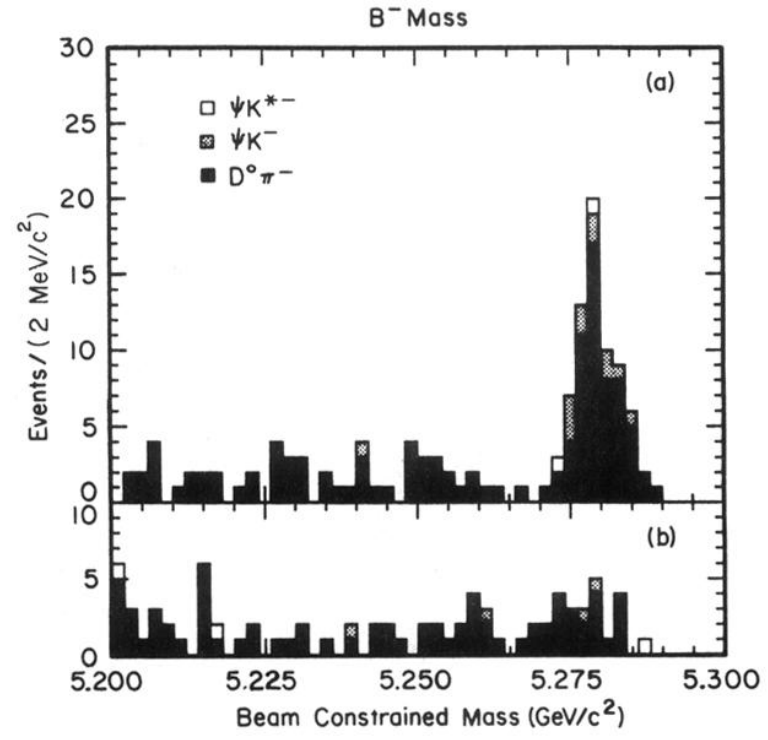

FIG. 9. Beam-constrained mass of reconstructed $B^{-}$candidates in modes used in the mass fit: (a) $|\Delta E|<2 \sigma_{E}$; (b) $2 \sigma_{E}<|\Delta E|<4 \sigma_{E}$. 